\title{
THE
}

\section{Instabilities in an Internal Solitary-like Wave on the Oregon Shelf}

Levin G. Lamb

David M. Farmer

University of Rhode Island, farmerd@uri.edu

Follow this and additional works at: https://digitalcommons.uri.edu/gsofacpubs

\section{Citation/Publisher Attribution}

Lamb, K. G., \& Farmer, D. (2011). Instabilities in an Internal Solitary-like Wave on the Oregon Shelf. Journal of Physical Oceanography, 41, 67-87. doi: 10.1175/2010JP04308.1

Available at: http://dx.doi.org/10.1175/2010JP04308.1

This Article is brought to you for free and open access by the Graduate School of Oceanography at DigitalCommons@URI. It has been accepted for inclusion in Graduate School of Oceanography Faculty Publications by an authorized administrator of DigitalCommons@URI. For more information, please contact digitalcommons-group@uri.edu. 


\title{
Instabilities in an Internal Solitary-like Wave on the Oregon Shelf
}

\author{
KEVIN G. LAMB \\ Department of Applied Mathematics, University of Waterloo, Waterloo, Ontario, Canada \\ DAVID FARMER \\ Graduate School of Oceanography, University of Rhode Island, Kingston, Rhode Island
}

(Manuscript received 1 July 2009, in final form 20 June 2010)

\begin{abstract}
Observations of internal solitary-like waves (ISWs) on the Oregon Shelf suggest the presence of KelvinHelmholtz billows in the pycnocline and larger-scale overturns at the back of the wave above the pycnocline. Numerical simulations designed to explore the mechanisms responsible for these features in one particular wave reveal that shear instabilities occur when (i) the minimum Richardson number $\mathrm{Ri}$ in the pycnocline is less than about 0.1 ; (ii) $L_{x} / \lambda>0.8$, where $L_{x}$ is the length of the unstable region with $\mathrm{Ri}<0.25$ and $\lambda$ is a half wavelength of the wave; and (iii) a linear spatial stability analysis predicts that $\ln \left(a_{f} / a_{i}\right)>\approx 4$, where $a_{i}$ and $a_{f}$ are the amplitudes of perturbations entering and leaving the unstable region. The maximum energy loss rate in our simulations is $50 \mathrm{~W} \mathrm{~m}^{-1}$, occurring at a frequency $8 \%$ below that with the maximum spatial growth rate.

The observations revealed the presence of anomalously light fluid in the center of the wave above the pycnocline. Simulations of a wave encountering a patch of light surface water were used to model this effect. In the presence of a background current with near-surface shear, the simulated ISW has a trapped surface core. As this wave encounters a patch of lighter surface water, the light surface water at first passes beneath the core. Convective instabilities set in and the light fluid is entrained into the core. This results in the formation of overturning features, which exhibit some similarities with the observed overturns.
\end{abstract}

\section{Introduction}

In this paper, we describe numerical simulations undertaken to explain observed instabilities in a large internal solitary-like wave (ISW) on the Oregon Shelf. ISWs are commonly observed in coastal regions of the world's oceans. They are predominantly generated by the nonlinear-dispersive evolution of internal tides generated by tidal flow over the shelf break or across ridges (Farmer et al. 2009), but other generation mechanisms include the relaxation of controlled flow over a sill (Farmer and Smith 1980), upstream influence of controlled flow over topography (Cummins et al. 2003; Stastna and Peltier 2004), near-resonant flow over topography (Bogucki et al. 1997), nonlinear interaction of an internal tidal beam with near-surface stratification (Gerkema 2001), adjustment of river plumes (Nash and Moum 2005),

Corresponding author address: Kevin G. Lamb, Department of Applied Mathematics, University of Waterloo, Waterloo ON N2L 3G1, Canada.

E-mail: kglamb@uwaterloo.ca and possibly Kelvin waves (Hosegood and van Haren 2004). ISWs are coherent, energetic events that can travel hundreds of kilometers. Wave-induced currents can resuspend sediments (Bogucki et al. 1997; Carter et al. 2005) and impact offshore engineering activities (Osborne and Burch 1980). Packets of ISWs can significantly affect acoustic transmission. They can also play an important role in mixing because of the large energy transport associated with their propagation.

There are at least four mechanisms through which ISWs can result in mixing. These include instabilities in the bottom boundary layer (Bogucki and Redekopp 1999; Stastna and Lamb 2002; Bogucki et al. 2005; Diamessis and Redekopp 2006; Stastna and Lamb 2008) or when waves break as they shoal (Klymak and Moum 2003; Vlasenko and Hutter 2002; Bourgault et al. 2007; Bourgault and Galbraith 2008). Two other mechanisms, the focus of this paper, include unstable wave cores (Lamb 2002; Carter et al. 2005) and shear instabilities in the fluid interior triggered by ISW-induced vertically sheared currents (Bogucki and Garrett 1993; Jeans and Sherwin 2001; Moum et al. 2003; Orr and Mignerey 


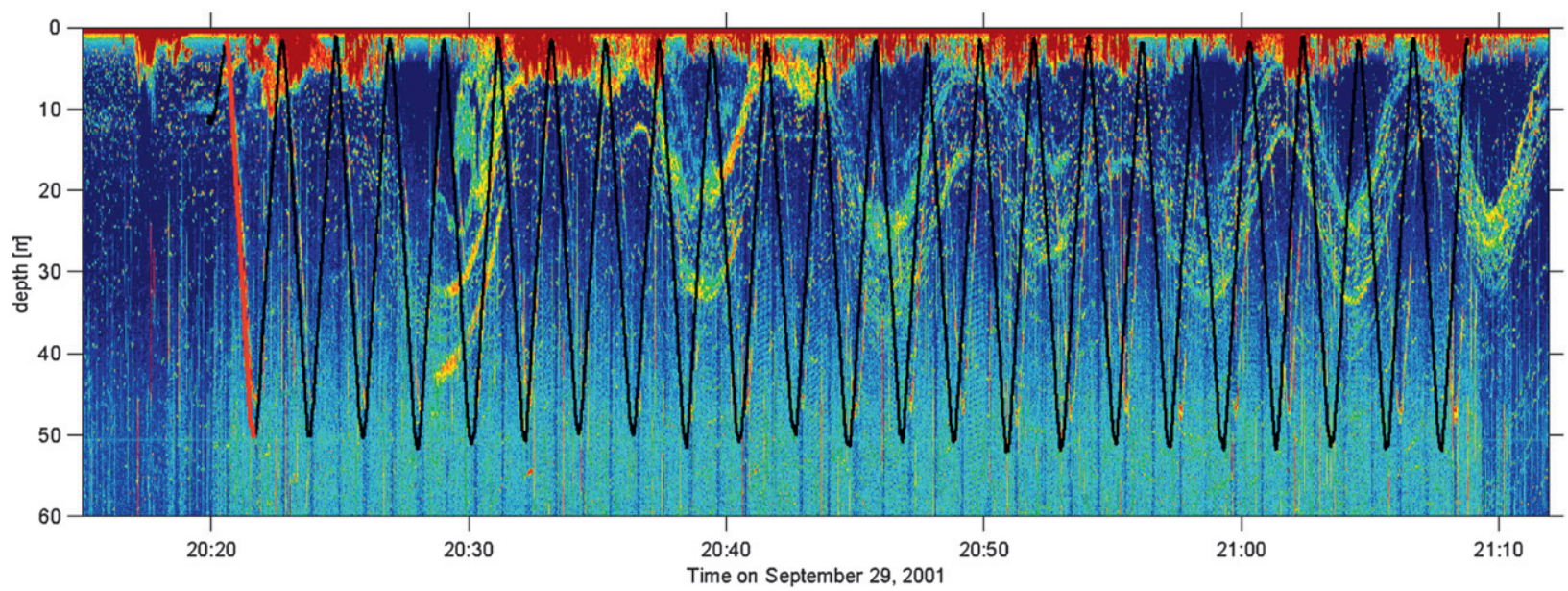

FIG. 1. Wave packet observed on Oregon Shelf. The wave is propagating from left to right. The sloping zigzag lines through the figure show the path of a CTD as it was raised and lowered through the fluid, giving high-resolution temperature and salinity measurements. The red colors indicate high levels of backscatter. Times are UTC.

2003; Fructus et al. 2009; Barad and Fringer 2010). Sandstrom and Elliott (1984) concluded that dissipation of ISWs constitutes the primary mixing mechanism inshore of the Scotian Shelf break. Holloway (1991) found that internal tides on the Australian North West Shelf provided all the mixing required to explain the temperature profile, and Jeans and Sherwin (2001) concluded that ISWs on the Portuguese Shelf provide an important energy source for mixing.

High-frequency acoustic images of ISWs in a variety of environments, including fjords (Farmer and Smith 1980), the Oregon Shelf (Moum et al. 2003), and the South China Sea (Orr and Mignerey 2003), show clear evidence of ISWs with shear instabilities in the pycnocline (e.g., Moum et al. 2003, Fig. 14). In the images acquired on the Oregon Shelf, such structures appeared intermittently. Figure 1 is an acoustic image of a packet of internal waves, the leading member of which is shown in greater detail in Fig. 2. These images, acquired during a collaborative study carried out from the R/V Wecoma, reveal several interesting characteristics discussed in detail below. The wave illustrated here, with $30-\mathrm{m}$ amplitude and $200-\mathrm{m}$ half-width, travels at about $0.6 \mathrm{~m} \mathrm{~s}^{-1}$ (based on GPS readings). The acoustic image also provides suggestive indications of instability and overturning and for this reason is selected for detailed analysis and simulation. The data presented here were acquired in the same area and during the same period as the observations discussed by Moum et al. (2003). Although no two images of unstable internal waves are identical, this example bears some similarity to others we have seen and thus provides a useful sample for detailed analysis.

Shear instabilities in progressive internal waves have been observed in the ocean (Woods 1968) and discussed by Thorpe (1968), who used the criterion that the Richardson number Ri must be less than $1 / 4$ for shear instabilities to occur. He recognized that the stability of short waves depends on the ratio of the wave period to the time scale associated with the growth of the instabilities. This was made more concrete by Troy and Koseff (2005), who analyzed laboratory experiments of progressive, periodic internal waves in a stratification consisting of a thin pycnocline separating two homogeneous layers of equal thickness. They used the TaylorGoldstein equation to predict temporal instability growth rates, arguing that the flow could be treated as parallel, provided that the wavelengths of the unstable modes were short compared to the wavelength of the waves. They found that waves were unstable, provided that $\bar{\sigma}_{i} T_{w}>5$, where $\bar{\sigma}_{i}$ is an average growth rate and $T_{w}$ is the length of time the instability remains in the patch of fluid with $\mathrm{Ri}<$ 0.25. Barad and Fringer (2010) obtained similar results for shear instabilities in numerical simulations of an ISW. Troy and Koseff found that a minimum Ri of (0.07-0.08) \pm 0.03 was required for overturning Kelvin-Helholtz $(\mathrm{KH})$ billows to form, whereas Barad and Fringer found that instabilities occurred if Ri fell below 0.1.

In laboratory experiments using a three-layer continuous stratification with two homogeneous layers separated by a linearly stratified layer, Fructus et al. (2009) also found a cutoff $\mathrm{Ri}$ of about 0.1 , although they observed unstable waves with $\mathrm{Ri}$ as high as 0.11 and stable waves with $\mathrm{Ri}$ as low as 0.087 . They found that $\sigma_{\max } T_{w}>5.4$ for their unstable waves, where $\sigma_{\max }$ is the maximum growth rate, and proposed a third instability criterion: namely, $L_{x} / \lambda>0.86$, where $L_{x}$ is the length of the region with $\mathrm{Ri}<0.25$ and $\lambda$ is the length of the region in which the vertical displacement of the base of the stratified layer exceeded half its maximal 


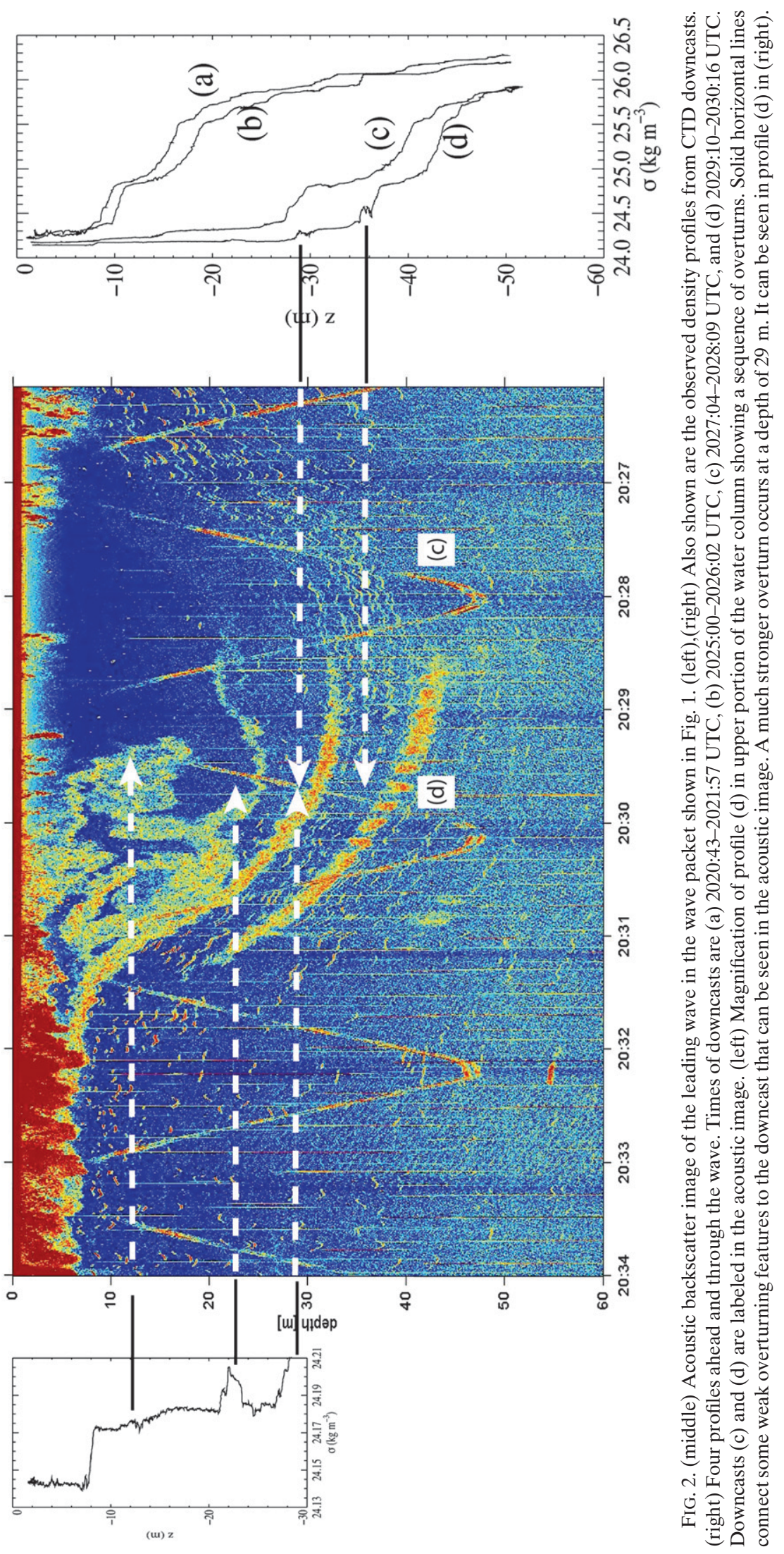


displacement. This criterion more cleanly separated their unstable and stable waves. Barad and Fringer found that $L_{x} / \lambda>0.86$ for all their unstable waves.

Convective instabilities, for which the fluid velocity exceeds the wave propagation speed, can also occur in internal waves. In numerical simulations of progressive internal waves, Fringer and Street (2003) found that convective instabilities occurred when $k \delta$ exceeded 0.56 , where $k$ is the wavenumber and $\delta$ a measure of the pycnocline thickness. Lamb $(2002,2003)$ reported on simulations of shoaling internal solitary waves of depression that broke via a convective instability as they shoaled provided the fluid was strongly stratified at the surface or there was near-surface shear. Carr et al. (2008) reported on mixed convective-shear instabilities in laboratory experiments using a continuous stratification consisting of a lower homogeneous layer and a linearly stratified upper layer. They performed experiments with both a free surface and with a fixed upper boundary and found that the results were sensitive to the surface boundary condition.

The purpose of this paper is to describe two-dimensional numerical simulations that seek to explain the unstable structures observed in Fig. 2 and to identify some of the conditions required for such instabilities to occur. Fringer and Street (2003) carried out two- and three-dimensional simulations of shear instabilities in progressive interfacial waves and found that three-dimensional processes do eventually become important and are crucial to simulating mixing; however, the initial instability is two dimensional. Barad and Fringer (2010) reported on one three-dimensional simulation of shear instabilities in an ISW, concluding that the primary instability was two dimensional, with three-dimensional instabilities occurring near the back of the wave. These results justify the use of two-dimensional simulations.

The paper is organized as follows: The observations leading to Fig. 2 are discussed in section 2. In section 3, the numerical model is described along with the background density and velocity fields used in the simulations. In section 4 , the method used to initialize the numerical model with ISWs is described. We first consider ISW solutions of the Dubreil-Jacotin-Long (DJL) equation and explore the sensitivity of these waves to the nearsurface shear in the background velocity. A limitation of this approach is that it does not yield unstable waves, motivating the development of a new method for initializing the model with unstable ISWs. Section 5 presents simulations of KH billows for cases with no background current. Comparisons are made with predictions of a spatial stability analysis. The energetics of the unstable waves is discussed in section 6 . In section 7 , we explore the overturning process in the core of the wave by extending our analysis to include the effects of spatial variability in the near-surface stratification, which might be expected offshore of the Columbia River estuary, where these measurements were made. The simulations make it clear that overturning in the core requires quite specific shear or near-surface stratification conditions. An interpretation of the observations in terms of the numerical simulations is summarized in section 8 .

\section{Observations}

The observations were acquired just southwest of the Columbia River plume $\left(46^{\circ} 7^{\prime} 15^{\prime \prime} \mathrm{N}, 124^{\circ} 16^{\prime} 45^{\prime \prime} \mathrm{W}\right)$, approximately $30 \mathrm{~km}$ west of the Oregon coast. The water depth shoals smoothly from the 100-m contour at the observational area to the $50-\mathrm{m}$ contour $16 \mathrm{~km}$ to the east. In addition to microstructure profiling (Moum et al. 2003), the instrumentation suite included a $300-\mathrm{kHz}$ hull-mounted ADCP; a 120-kHz narrow-beam echo sounder with a half beamwidth of $3^{\circ}$; and a CTD, which was profiled continuously. The ADCP was sampled using 2-m vertical bins and 5-s averages. The vertical resolution was insufficient to measure the narrow wave-induced shear layer across the pycnocline in the ISW, but it did provide some information on the weak background currents at depths greater than $8 \mathrm{~m}$, beyond the interference due to reverberation from the hull. This range limitation prevented us from resolving the near-surface shear, which can result in the generation of trapped vortical cores.

Packets of ISWs were observed propagating inshore on a semidiurnal basis. Figure 1 shows the packet from which we selected the leading wave (Fig. 2). Detailed measurements using the CTD were typically acquired with the ship slowly advancing through the waves in the opposite direction to their propagation, following which the vessel ran ahead of the waves at 6-8 kt to sample the waves successively as they advanced eastward. The echo sounder acquired images of the acoustical target strength of biota and microstructure. No attempt is made here to separate these different contributions, but numerous applications of this approach tend to support the view that there is a tendency for the biological scatter to become organized along density steps. Our simultaneous CTD and acoustical images are consistent with this interpretation. The CTD was continuously profiling the upper $45 \mathrm{~m}$ during passage of the wave and can be readily identified in the acoustic image, providing a useful guide for relating density profiles to the acoustic images. The CTD is deployed just aft of the echo sounder and is subject to a small but unmeasured wire angle due to the forward motion of the vessel. Prior to arrival of the waves, the stratification is concentrated in the upper $25 \mathrm{~m}$, below which the density profile has a nearly constant gradient of $0.0183 \mathrm{~kg} \mathrm{~m}^{-4}$. The wave is traversed in approximately 
$5 \mathrm{~min}$, requiring rapid tow-yo profiles to sample the wave structure. There were typically three downcast profiles per wave.

Figure 2 (right) shows four $\sigma$ density profiles taken from CTD downcasts $\left(\sigma=\rho-1000 \mathrm{~kg} \mathrm{~m}^{-3}\right.$, where $\rho$ is the density). Profiles (a) and (b) are taken well ahead and near the leading edge of the wave. Profiles (c) and (d) are in the central and rear portion of the wave. The corresponding downcasts are labeled in the echo-sounder image. Profiles (a) and (b) show that the broad 20-m-thick pycnocline includes some important finescale features, particularly two sharp pycnoclines at depths of about 9 and $15 \mathrm{~m}$. These are the layers in which instabilities occur in the numerical simulations. Moum et al. (2003) observed increased scattering along density steps similar to these and explained it in terms of enhanced acoustic target strength due to shear instability and microstructure. Periodic fluctuations in target strength along these two density steps are almost certainly modulated by waveinduced motion of the ship and do not represent individual overturning structures.

The density profiles also show unstable segments associated with the two sharp pycnoclines, especially in profile (d). The upper portion of this profile is shown with an expanded density scale in the left panel of Fig. 2. This reveals some weakly unstable structures at depths of 4 and $12 \mathrm{~m}$, a broader weak overturn between depths of 15 and $20 \mathrm{~m}$, and a much stronger overturn at a depth of $22 \mathrm{~m}$ (the overturn strength $\Delta \rho$ is the difference in the maximum and minimum density values in the overturn). Larger overturns can be seen at 29- and 35-m depths in the right panel. Horizontal dashed lines are included to aid in relating these features to the corresponding portion of the image. Small discrepancies in the depths are to be expected as the CTD is displaced from the sonar location.

Details on the five most significant overturns in profile (d) are given in Table 1. Before analyzing the density profile, it was smoothed by taking a weighted average of five consecutive density values (weights 0.5 for the middle value and 0.125 for the neighboring values). The overturning region is the smallest region such that all the fluid above (below) is lighter (denser) than the fluid in the overturn (Galbraith and Kelley 1996). The table includes the range of depths spanned by the overturn, overturn thickness, overturn strength, and the available potential energy (APE). Values were interpolated onto a uniformly spaced grid using cubic splines before calculating the APE. The smoothing reduces the thickness of the overturn, $\Delta \rho$, and the APE, so these values only provide a rough guide. Some small overturns that can be seen in the density profile (e.g., that at a depth of $7.5 \mathrm{~m}$ in the left panel of Fig. 2) fail the run length test of Galbraith and Kelley (1996). Following Galbraith and Kelley (1996),
TABLE 1. Values quantifying the strength of the largest five overturns (based on APE) in density profile (d) of Fig. 2. The first column gives range of depths spanning the overturn, the second column is the thickness of the overturning region, $\Delta \rho$ is the difference between the maximum and minimum density in the overturn, and APE is the APE of the overturn.

\begin{tabular}{cccc}
\hline \hline Overturn range $(\mathrm{m})$ & Thickness $(\mathrm{m})$ & $\Delta \rho\left(\mathrm{kg} \mathrm{m}^{-3}\right)$ & APE $\left(\mathrm{J} \mathrm{m}^{-2}\right)$ \\
\hline$(-1.6,-7.8)$ & 6.2 & 0.01 & 0.15 \\
$(-8.3,-14.0)$ & 5.6 & 0.01 & 0.02 \\
$(-15.0,-28.1)$ & 13.1 & 0.03 & 1.24 \\
$(-28.6,-30.4)$ & 1.8 & 0.05 & 0.08 \\
$(-35.2,-36.3)$ & 1.2 & 0.08 & 0.14 \\
\hline
\end{tabular}

we also searched for loops in the temperature-salinity (TS) diagram. The only one found was at a depth of 35$36 \mathrm{~m}$. Because this overturn is clearly visible in both the temperature and salinity profiles, with a consistent structure in both form, thickness and depth, we consider this to be a true overturn. The presence of the loop suggests the values of $\Delta \rho$ and APE may have large errors; however, for our purposes, it is the presence and approximate thickness of this overturn which is of interest. We have not computed Thorpe scales, because the CTD is passing through the wave as it moves past the ship, so we are looking at an $x-z$ profile in a rapidly evolving background flow. For this reason, we do not have a statistically stationary signal, because different parts of the heterogeneous macrostructure are sampled by the CTD as it descends.

Density profiles (c) and (d) in Fig. 2 show the presence of near-surface fluid, which is lighter (fresher and warmer) than any detected upstream of the wave. This is more clearly illustrated in Fig. 3, where the anomalously low density values are shaded. This suggests that the wave contains a core of light fluid, possibly because of the presence of patches of light surface water associated with the Columbia River plume. The lack of near-surface current observations precludes a direct check that waveinduced currents exceed the wave propagation speed; however, the presence of lighter fluid is suggestive and motivates exploration with numerical simulations, which are discussed in section 7 .

\section{The numerical model}

Simulations are carried out with a two-dimensional nonhydrostatic model (Lamb 1994, 2007), which uses the Boussinesq approximation and a rigid lid at the surface $z=0$. Rotation is not included because of the small time and length scales of interest. The model equations are

$$
\rho_{0} \frac{D \mathbf{u}}{D t}=-\nabla p-\rho g \mathbf{k}+(0, F)
$$




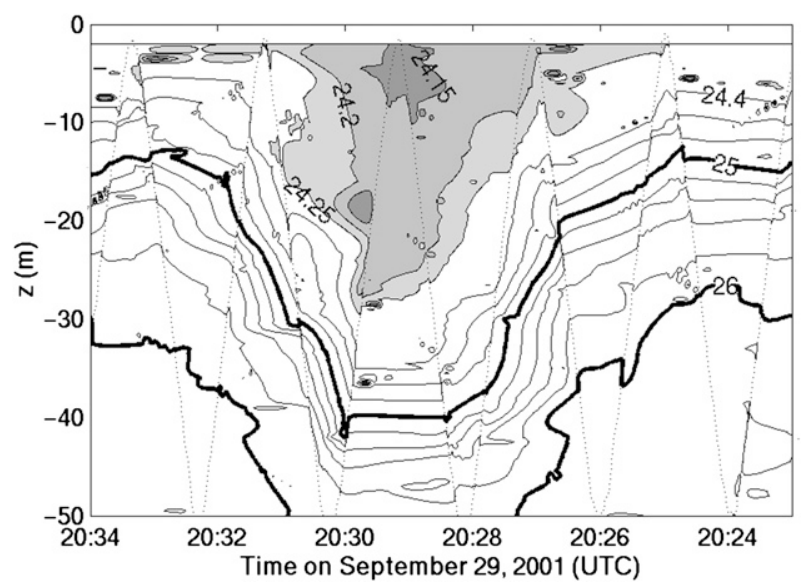

FIG. 3. Observed density field $\sigma=\rho-1000 \mathrm{~kg} \mathrm{~m}^{-3}$ ahead of the wave interpolated from CTD measurements obtained along the zigzagged lines. The shaded region indicates fluid lighter than any observed ahead of the wave. This is a contour plot of the raw, sparse density data and hence many of the overturns are artifacts. It is only shown to illustrate the patch of light fluid.

$$
\begin{aligned}
\frac{D \rho}{D t} & =0, \quad \text { and } \\
\nabla \cdot \mathbf{u} & =0,
\end{aligned}
$$

where standard terminology has been used, with $\mathbf{u}=(u, w)$ being the two-dimensional velocity field. The model is initialized with an unstable ISW (section 4). The assumption of a constant depth is consistent with the gradual slope of the Oregon Shelf, allowing us to do the computations in a reference frame moving with the ISW, which propagates to the right through the fluid. In the horizontal, 6000 grid points gave a horizontal resolution of $0.167 \mathrm{~m}$ over a domain $1000 \mathrm{~m}$ long centered on the wave. A stretched vertical grid, using 450 grid points, provided a vertical resolution of $1.3 \mathrm{~m}$ in the lower $40 \mathrm{~m}$ and $0.13 \mathrm{~m}$ in the upper $50 \mathrm{~m}$, with a smooth transition in between. Viscosity and diffusion are ignored because of the high Reynolds number of the ISW under investigation $\left[O\left(10^{6}\right)\right.$ based on wave-induced current and pycnocline thickness]. Hogg and Ivey (2003) found that, for a Reynolds number of 100, two-dimensional shear instabilities were very similar to inviscid instabilities. Viscosity and diffusion would be required in threedimensional simulations of mixing.

Although the flow within the wave is hydrodynamically unstable, growth of instabilities do not occur without small perturbations, which will normally be present in the active upper-ocean environment. We used a forcing function $F$ in the vertical momentum equation to generate predominately mode-one perturbations upstream of the wave. This function,

$$
F(x, z, t)=\rho_{0} G(t) e^{-[(x-450) / 10]^{2}}\left\{e^{-[(z+9) / 4]^{2}}+e^{-[(z+15) / 4]^{2}}\right\},
$$

is comprised of the sum of two terms, centered in each sharp pycnocline upstream of the wave. The forcing exceeds $10 \%$ of its maximum value between depths of about 4 and $22 \mathrm{~m}$. For most runs, the time dependence is a single harmonic,

$$
G(t)=a \sin \left(\omega_{f} t\right)
$$

allowing us to explore the frequency response of the system. A few runs were carried out using a "random" forcing consisting of the sum of 96 different harmonics with frequencies ranging between 0.01 and $0.2 \mathrm{~s}^{-1}$ of the form

$$
G(t)=\sum_{n=0}^{n=95} a_{n} \sin \left[(0.01+0.002 n) t+\phi_{n}\right]
$$

where the amplitudes $a_{n} \in\left(0, a_{\max }\right)$ and phases $\phi_{n}$ were randomly generated. The random forcing function is shown in Fig. 4 for $a_{\max }=10^{-5} \mathrm{~m} \mathrm{~s}^{-2}$.

Because the forcing term is used in a reference frame fixed with the wave, the frequency of the forcing in a reference frame that moves with the fluid is Doppler shifted. The horizontal wavenumbers $k$ of the forced small amplitude waves are given by

$$
\omega_{f}= \pm \Omega_{n}(k)-c k
$$

where $c$ is the propagation speed of the ISW relative to the fluid and $\Omega_{n}(k)>0$ is the frequency of a mode- $n$ wave in a reference frame fixed with the fluid ahead of the wave. Choosing $\omega_{f}>0$, we have $k<0$, because for the supercritical propagation speeds of ISWs $\left|\Omega_{n}(k) / k\right|<c$. There are two wavenumbers with $k<0$ satisfying (7); hence, the forcing generates two different linear waves for each vertical mode. The shorter mode-one wave, which propagates rightward relative to the fluid, dominates the response.

\section{Model stratification and background velocity}

A few stratifications were used in the model simulations. The base stratification,

$$
\begin{aligned}
\bar{\sigma}_{1}= & 25.2550-0.290 \tanh \left(\frac{z+9.0}{d_{1}}\right) \\
& -0.35 \tanh \left(\frac{z+15.5}{d_{2}}\right)-0.375 \tanh \left(\frac{z+30.0}{9.0}\right) \\
& +0.15 \operatorname{sech}\left(\frac{z+24.0}{3.0}\right)
\end{aligned}
$$



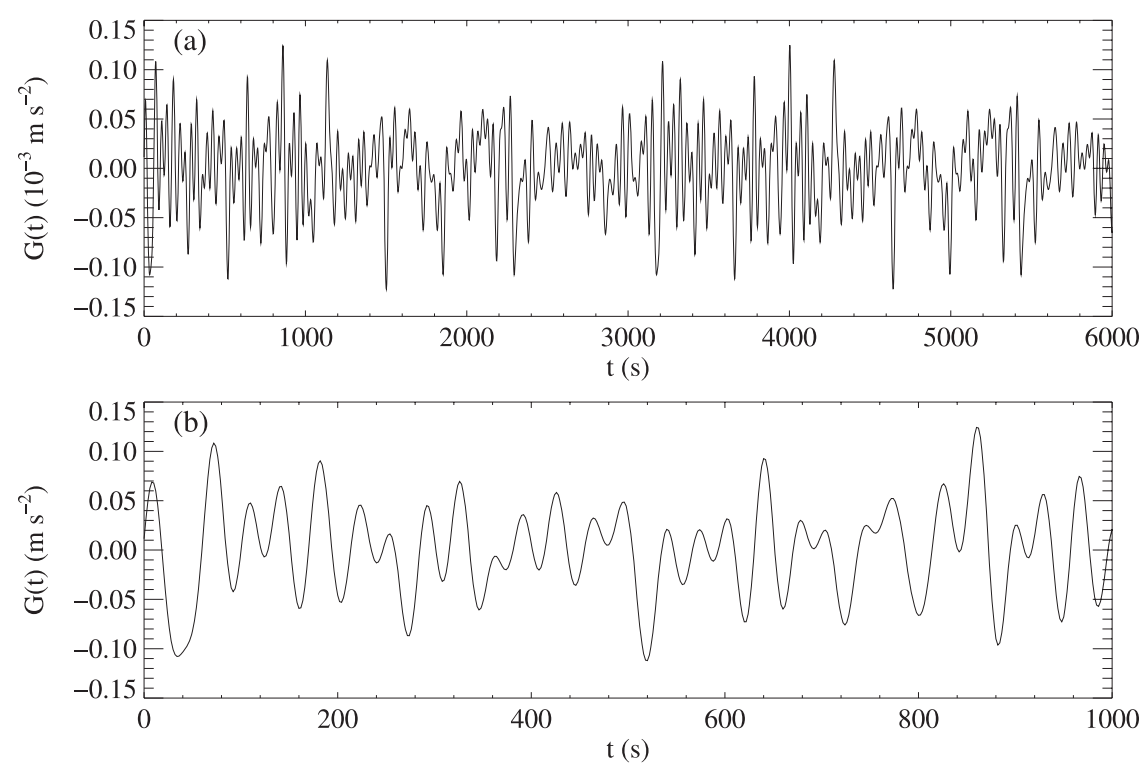

FIG. 4. (a) Time series of the random forcing $G(t)$ for $a_{\max }=10^{-5} \mathrm{~m} \mathrm{~s}^{-2}$.

(b) The first $1000 \mathrm{~s}$.

with

$$
\left(d_{1}, d_{2}\right)=(1.0,1.5) \mathrm{m}
$$

is shown in Fig. 5, where it is compared with an observed density profile upstream of the wave. The observed profile shows the presence of small-scale overturns, for example at depths of about 3 and $7 \mathrm{~m}$, which appear to be real, having signals in both the temperature and salinity data spanning approximately 50 and 20 data points, respectively. These particular features are not seen in adjacent density profiles, which have small overturns at other depths.

In the absence of a background current, $d_{2}<0.5 \mathrm{~m}$ was required for shear instabilities to be generated in the lower sharp pycnocline. This produced a much sharper pycnocline than observed, suggesting that the presence of shear instabilities on this pycnocline in the observations may be associated with a background shear across it. Some simulations were carried out in which the density was reduced by $0.1 \mathrm{~kg} \mathrm{~m}^{-3}$ in the upper $3 \mathrm{~m}$, motivated by the observed low density values in the center of the wave. This modification did not significantly affect the results (not shown).

We extrapolated the current profile up to the surface using four different profiles (Fig. 6), with the results being not particularly sensitive to the velocity profile below 60 $\mathrm{m}$ depth. Properties of large ISWs vary considerably as the near-surface shear is changed (see below).

\section{Model initialization}

The numerical model was initialized with a single ISW calculated by solving the DJL equation (Stastna and
Lamb 2002; Lamb 2003). For background currents $\bar{U}_{3,4}$, large waves may have a core with closed streamlines. For the current application, the cores do not contain anomalously light fluid, because our density profiles have a constant density in a surface mixed layer.

Figure 7 shows ISWs obtained by solving the DJL equation for the four different background currents. These waves have either an amplitude of $35 \mathrm{~m}$ (Figs. 7a,d)

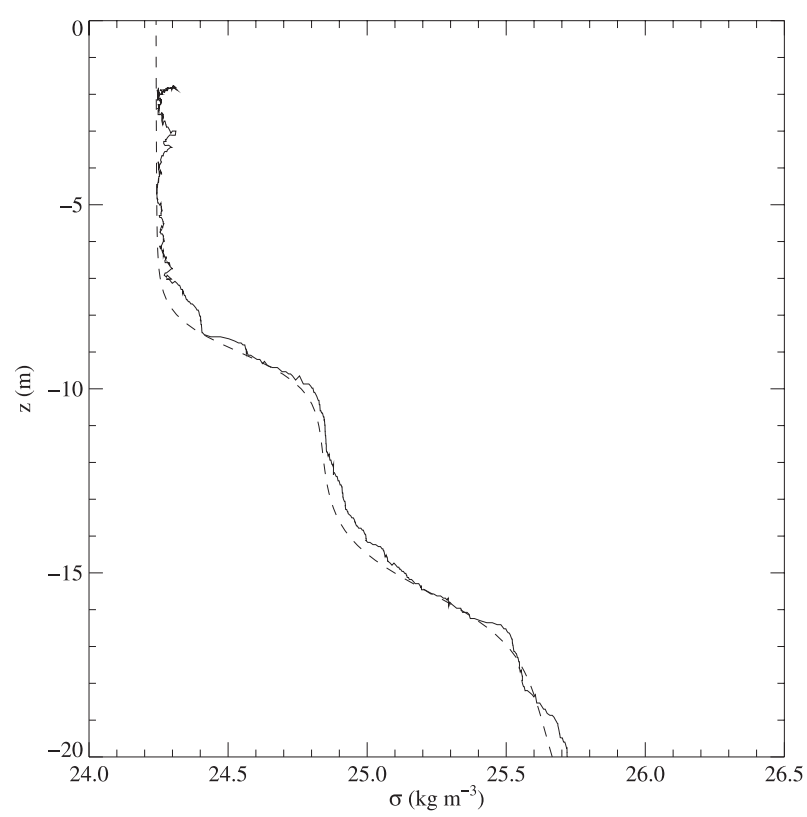

FIG. 5. Model stratification (dashed line) and an observed density profile ahead of the wave (solid line). 


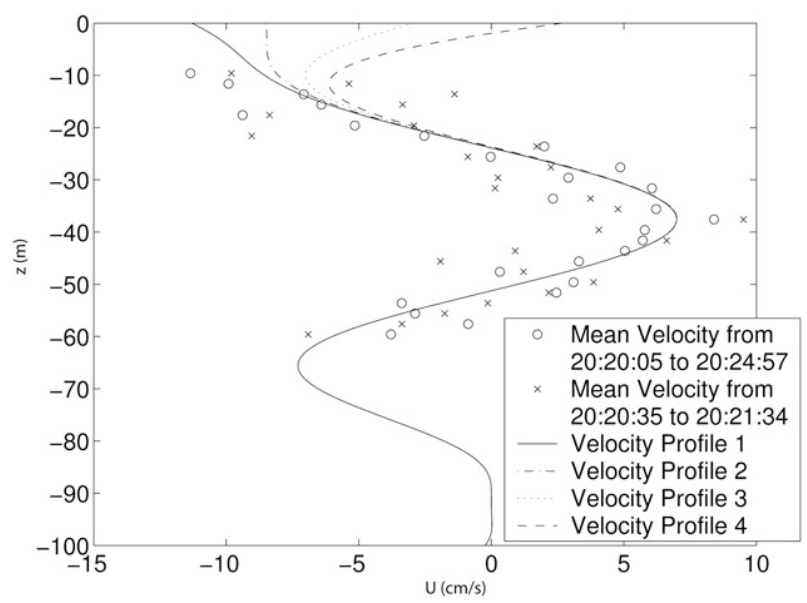

FIG. 6. Model velocity profiles. Positive values are flow in the direction of wave propagation.

or are the largest waves that could be directly calculated (Figs. 7b,c). The wave obtained using $\bar{U}(z)=\bar{U}_{4}(z)$ has a core. The symbols indicate the estimated locations of density values $\sigma=24.8$ and $25.5 \mathrm{~kg} \mathrm{~m}^{-3}$ (corresponding to the bottom of the two sharp pycnoclines) taken from the CTD downcasts with start times of 2025, 2027, and 2029 UTC (see Fig. 3). The relative horizontal location of the observed values was estimated using the GPSbased ship speed of $0.21 \mathrm{~m} \mathrm{~s}^{-1}$ and the calculated wave propagation speed of $0.63 \mathrm{~m} \mathrm{~s}^{-1}$. The location of the rightmost observation was chosen to give a reasonable fit to the computed waves. This conversion is subject to uncertainty but does facilitate comparisons between different waves and demonstrates similarity between the simulations and density profile observations.

The amplitude and width of the observed wave is captured reasonably well with a 35-m wave for any of the background currents used. What differs is the flow in the center of the wave above the pycnocline (e.g., the occurrence of a core) and the Richardson numbers in the pycnoclines, which have a direct bearing on the occurrence of shear instability.

Profiles of horizontal velocity down the center of computed waves with amplitude $30 \mathrm{~m}$ are shown in Fig. 8 . They are virtually identical in the lower $60 \mathrm{~m}$ but have significant differences in the upper $30 \mathrm{~m}$. In the upper mixed layer, baroclinic vorticity generation is negligible and hence vorticity is preserved as the fluid in this layer
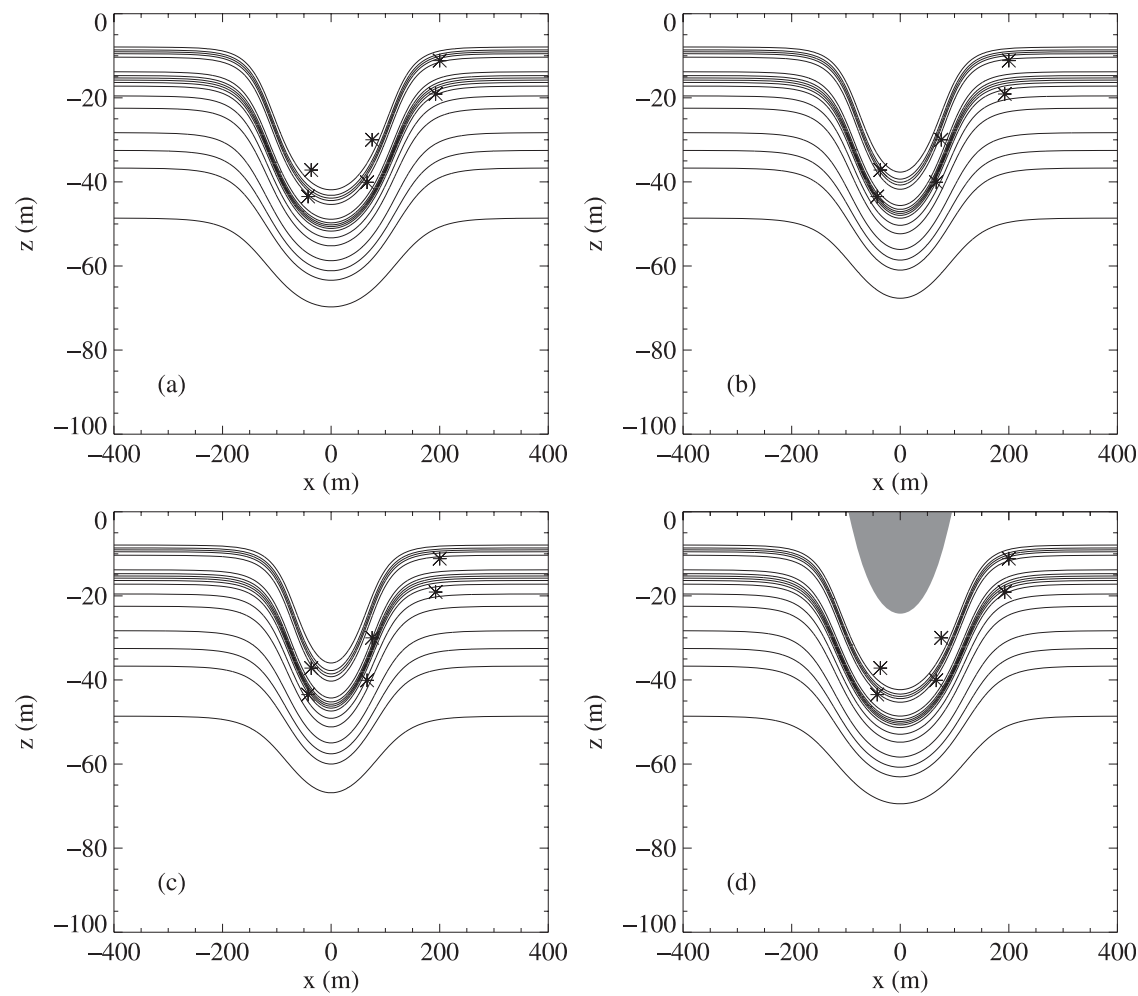

FIG. 7. Density contours for waves of amplitude $35 \mathrm{~m}$ or waves of maximal amplitude for the four background currents. Symbols indicate estimated locations of density values $\rho=1024.8$ and $1025.5 \mathrm{~kg} \mathrm{~m}^{-3}$ as calculated from CTD downcasts. (a) $\bar{U}=\bar{U}_{1}$ : amplitude $35.1 \mathrm{~m}$. (b) $\bar{U}=\bar{U}_{2}(z)$ : amplitude $31.8 \mathrm{~m}$. (c) $\bar{U}=\bar{U}_{3}(z)$ : amplitude $30.5 \mathrm{~m}$. (d) $\bar{U}=\bar{U}_{4}(z)$ : amplitude $34.9 \mathrm{~m}$. The shaded region is the wave core. 

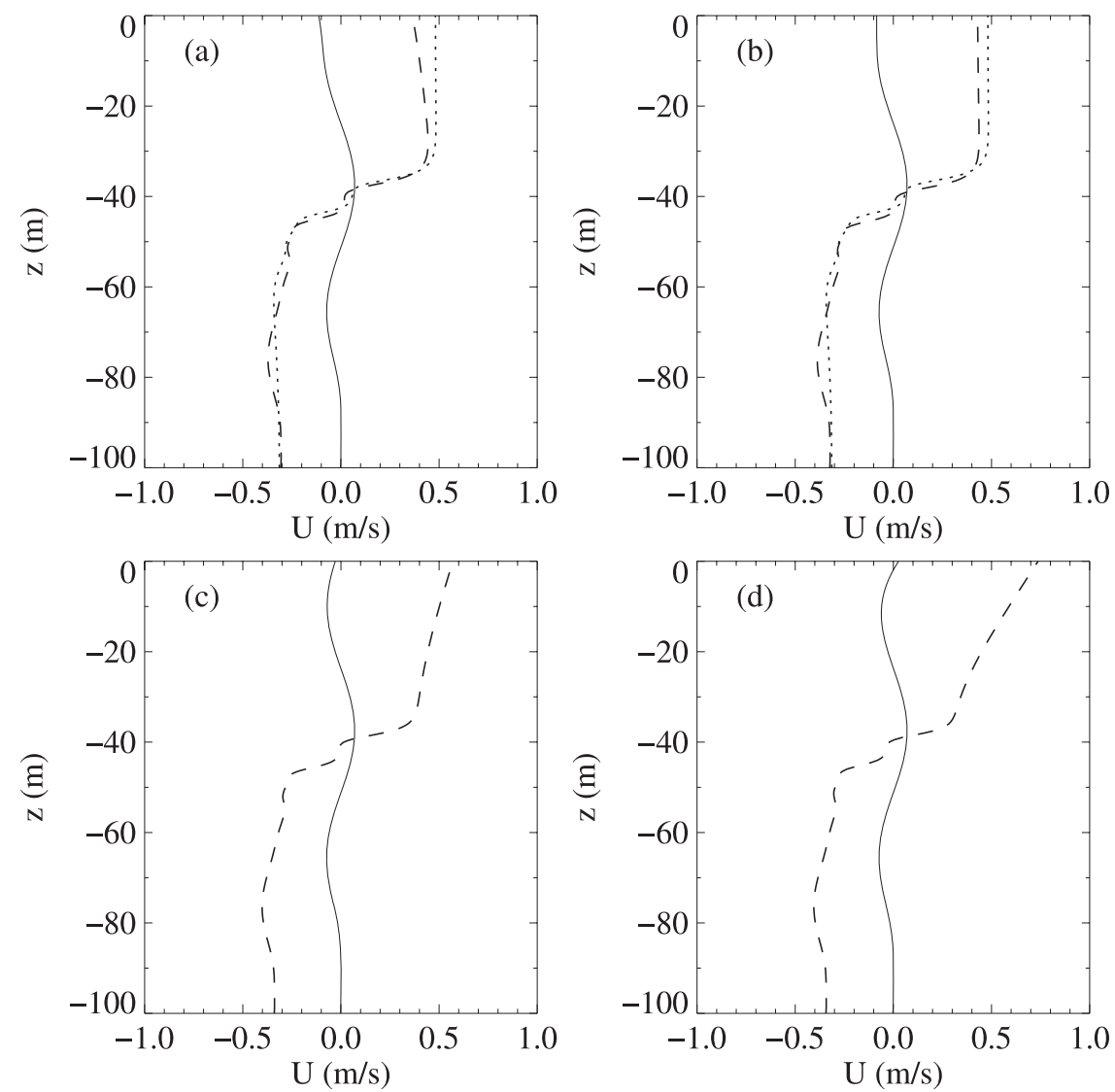

FIG. 8. Velocity profiles down the center of rightward-propagating ISWs of amplitude $30 \mathrm{~m}$ using density profile 1 : (a) $\bar{U}_{1}(z)$, (b) $\bar{U}_{2}(z)$, (c) $\bar{U}_{3}(z)$, and (d) $\bar{U}_{4}(z)$. The solid curve is the background velocity profile, and the dashed curve is the profile in the wave. In (a),(b), the velocity profile for a wave of amplitude $28 \mathrm{~m}$ computed with no background flow is shown with dotted lines for comparison.

enters the wave and is stretched vertically from a thickness of about 8 to $30 \mathrm{~m}$. This results in the much greater surface velocities for $\bar{U}=\bar{U}_{3,4}$. For velocity $\bar{U}_{4}$, this results in surface currents that exceed the wave propagation speed as required for the formation of a wave core (Lamb 2003).

For the stratification and background currents of interest here, the largest waves that can be computed directly had Richardson numbers of about 0.12 , and none of them exhibited KH instabilities in time-dependent simulations. To obtain waves with a lower Ri, we first computed a large wave with an alternative background velocity profile $\bar{U}_{\text {alt }}$ and/or stratification $\bar{\rho}_{\text {alt }}$. Next, we changed the horizontal velocity by subtracting $\bar{U}_{\text {alt }}$ and adding the desired background flow. Using the timestepping model to allow this wave to adjust to the new background fields yielded a steady ISW. During the adjustment process, the density field can be modified by changing the stratification at the inflow boundary. This adjustment, during which there is little change in the wave amplitude, took approximately one hour, which has significant implications for the behavior and propagation of waves in changing topography and stratification. The wave propagation speed changed slightly.

Comparisons of the velocity down the center of an adjusted wave using $\bar{U}=0$ (wave 1 in Table 2) with observations are presented in Fig. 9a. The observed profile near the center of the wave at time 2028:25 UTC (see Fig. 2) is shown along with the mean velocity between 2028:05 and 2028:45 UTC (average of nine profiles). For comparison, the average of the observed velocities ahead of the wave between 2020:05 and 2024:57 UTC (see Fig. 6) is included. As expected, the simulated velocity profile is vertically uniform above the pycnocline and has strong shear layers across the two sharp pycnoclines (vorticity maximum of $0.14 \mathrm{~s}^{-1}$ ). In contrast, the observed velocity profile is almost linear with a mean vorticity of about $0.02 \mathrm{~s}^{-1}$, and it lacks strong shear layers across the pycnocline. The velocity change between depths of 10 and $60 \mathrm{~m}$ is similar to that in the simulated wave. The lack of a strong shear layer at the depth of 
TABLE 2. Initial waves for simulations of shear instabilities: no background current and $\left(d_{1}, d_{2}\right)=(1.0,1.5)$. Here, max $|\eta|$ is the wave amplitude (maximum isopycnal displacement); $c$ and $\delta c$ are the background current at the surface outside the wave and the wave propagation speed in the computational domain; $\mathrm{Ri}_{1}$ and $\mathrm{Ri}_{2}$ are the minimum Richardson numbers in the upper and lower sharp pycnoclines, respectively; $a_{f} / a_{i}$ is the ratio of the final to initial amplitudes, predicted by linear stability theory, of a perturbation as it passes through the region with $\mathrm{Ri}_{1}<0.25$; and $L_{x} / \lambda$ is the ratio of the length of the region with $\mathrm{Ri}<0.25$ (upper sharp pycnocline) to the half wavelength $\lambda$ of the ISW (see text).

\begin{tabular}{ccccccccc}
\hline \hline Wave & $\max |\eta|(\mathrm{m})$ & $c\left(\mathrm{~m} \mathrm{~s}^{-1}\right)$ & $\delta c\left(\mathrm{~m} \mathrm{~s}^{-1}\right)$ & $\mathrm{Ri}_{1}$ & $\mathrm{Ri}_{2}$ & $\ln \left(a_{f} / a_{i}\right)$ & $L_{x} / \lambda$ & $\mathrm{Unstable}$ \\
\hline 1 & 33.9 & 0.635 & -0.0003 & 0.089 & 0.197 & 7.0 & 1.07 & Yes \\
2 & 32.2 & 0.631 & -0.0008 & 0.095 & 0.211 & 5.7 & 0.87 & Yes \\
3 & 29.8 & 0.628 & -0.0005 & 0.110 & 0.236 & 3.8 & 0.80 & Marginal \\
4 & 28.2 & 0.623 & 0 & 0.117 & 0.252 & 3.1 & 0.75 & - \\
\hline
\end{tabular}

the pycnocline indicates that the velocity observations are not of sufficient quality to make predictions of the potential for shear instabilities. In Fig. 9b, the simulated velocity profile using background velocity $\bar{U}_{4}$ is compared with that in the wave using no background velocity for the 36-m wave used below in our simulation of a wave with a core encountering a surface pool of light fluid. The shear above the pycnocline for this wave is similar to the observed shear.

Figure 10 shows the Richardson number profile down the center of the adjusted wave. The Richardson number now has a minimum value of 0.089 in the upper pycnocline and 0.197 in the lower pycnocline. Figure 11 overlays Richardson number contours on the density
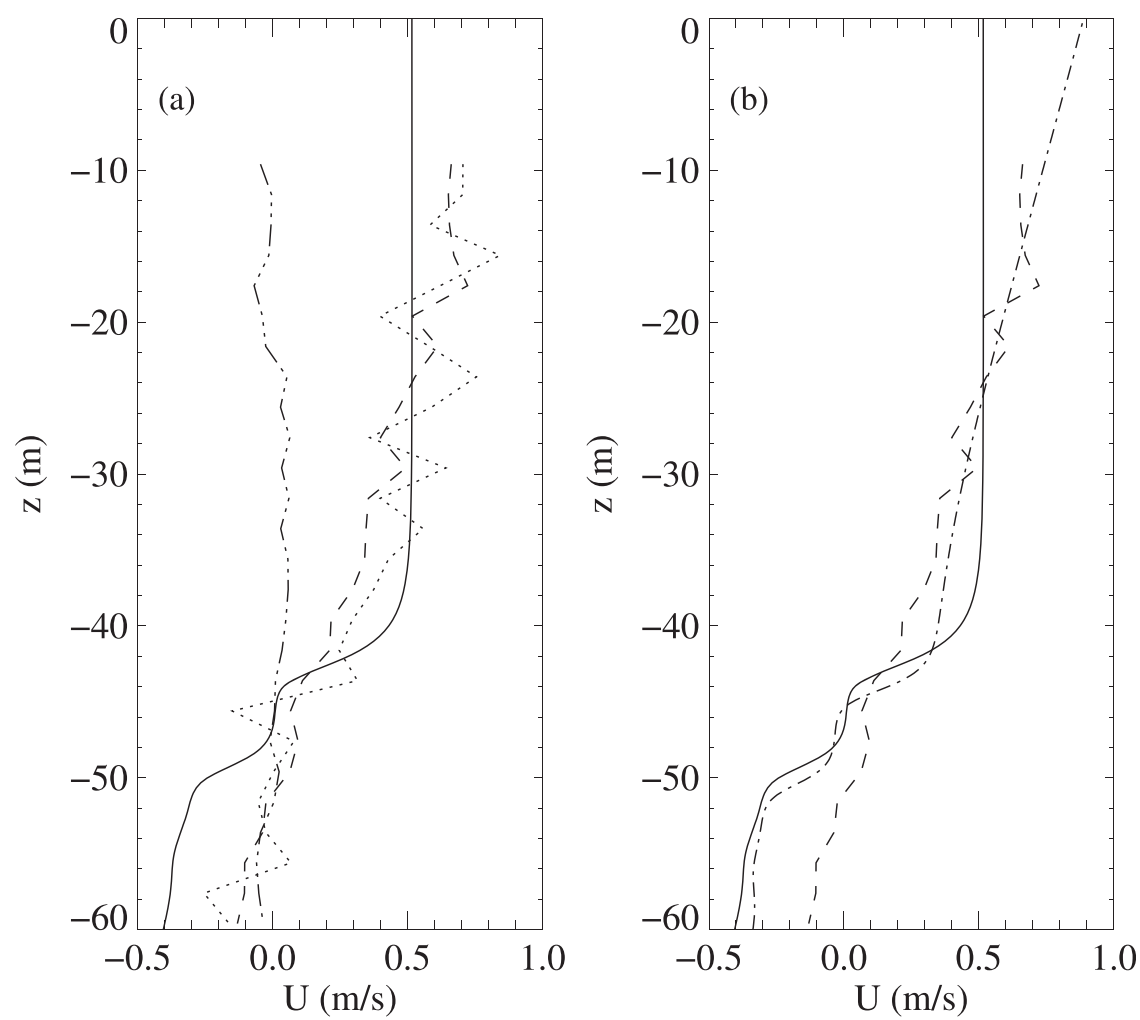

FIG. 9. Simulated and observed horizontal velocity and profiles. (a) The solid line is simulated horizontal velocity in the reference frame fixed with the fluid ahead of the wave for an adjusted 34-m wave; the dotted line is the observed profile near the center of the wave (time 2028:25 UTC); the dashed line is observed velocities averaged between 2028:05 and 2028:45 UTC; and the dashed-dotted line is observed velocity ahead of the wave, averaged from 2020:05 to 2024:57 UTC. (b) Dashed-dotted line is the simulated 36-m wave using $U_{4}(z)$ (the same wave as in Fig. 21); the other curves are as in (a). 


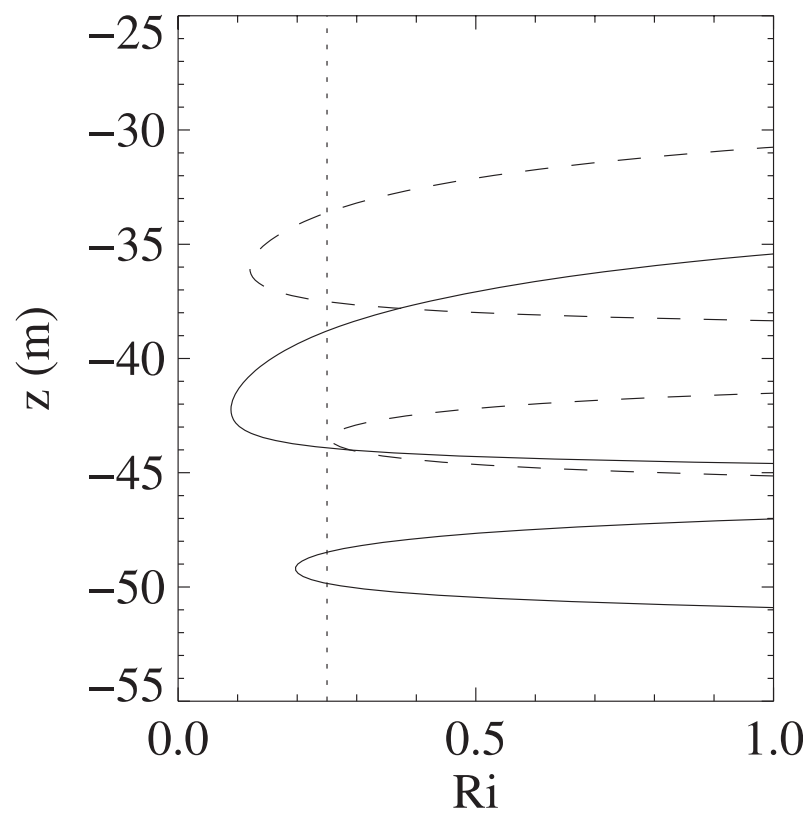

FIG. 10. Richardson number profile down the center of wave across the pycnocline: no background current, $\left(d_{1}, d_{2}\right)=(1.0,1.5)$ $\mathrm{m}$. The solid line is an adjusted wave of amplitude $33.9 \mathrm{~m}$ obtained by computing an initial wave of amplitude $35 \mathrm{~m}$, subtracting the background current and allowing the wave to adjust (case 1). The dashed line is a wave of amplitude $28.2 \mathrm{~m}$ (case 4).

field. There is a thin band, less than $2 \mathrm{~m}$ thick and about $80 \mathrm{~m}$ in length, in which $\mathrm{Ri}<0.1$.

\section{Simulations of shear instabilities}

For simplicity, we only discuss simulations without a background current. Properties of four initial waves are provided in Table 2. A large number of simulations have been carried out using the three largest of these waves (Table 3). For the most part, the sinusoidal forcing function (5) was used, enabling us to explore the frequency response of the system and to make comparisons with the predictions of linear stability theory. A few simulations were done with the random forcing function (6) using the largest wave only. Figure 12 shows the minimum Richardson number in the upper sharp pycnocline as a function of $x$ for these waves. Although the minimum Richardson number decreases slightly with increasing wave amplitude, the length of the region with near minimum Richardson numbers increases notably. This length is very important, because any unstable perturbations need time to grow (Thorpe 1968; Troy and Koseff 2005). Values of $L_{x} / \lambda$ for our waves were calculated using an isopycnal at the base of the upper sharp pycnocline to determine $\lambda$.

Information on the individual simulations is given in Table 3. Instabilities were easily generated in the two

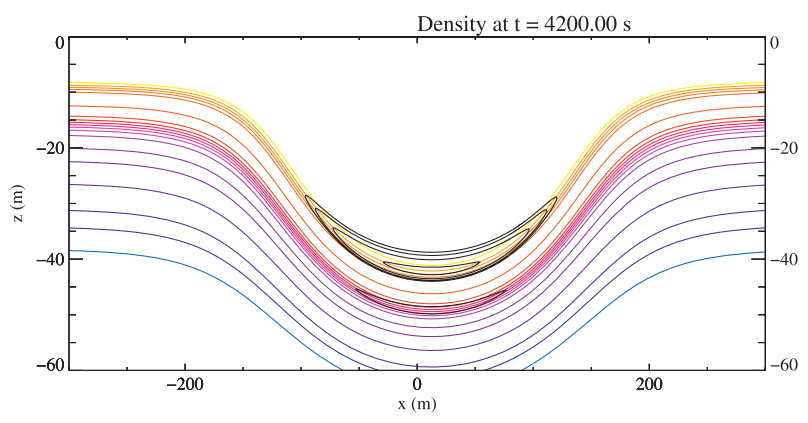

FIG. 11. Initial wave 1 (see Table 2). The color lines are density contours, and the black lines are Richardson number contours $(\mathrm{Ri}=0.25,0.2,0.15,0.1)$. This is the same wave as in Fig. 10 .

largest waves, which have $L_{x} / \lambda=0.87$ and 1.07. Figure 13 shows results for four forcing frequencies using the largest wave. The perturbations cannot be detected until the center of the wave, after which they grow rapidly in the upper sharp pycnocline. KH billows are formed for forcing frequencies between about 0.04 and $0.14 \mathrm{~s}^{-1}$. At $\omega_{f}=0.04 \mathrm{~s}^{-1}$, the unstable waves did not turn over until about $x=200 \mathrm{~m}$, at which point the billows were spaced $100 \mathrm{~m}$ apart. At $\omega_{f}=0.03 \mathrm{~s}^{-1}$, the perturbations grew enough to be detected downstream of the wave center but no rollups occurred. No growth of the perturbation could be detected for $\omega_{f}=0.16 \mathrm{~s}^{-1}$ at the scale of Fig. 13. The dependence of the instability on the amplitude of the ISW is illustrated in Fig. 14. The results for the two largest waves are similar, whereas for the $29.8 \mathrm{~m}$ wave $\left(L_{x} / \lambda=0.80\right)$ perturbations are barely detectible. Hence, no simulations were carried out with the smallest wave. Based on these results, the critical value of $L_{x} / \lambda$ separating stable from unstable waves is about 0.8 . This is $7 \%$ less than the value of 0.86 reported by Fructus et al. (2009) for a different stratification.

To provide confidence in our simulations of smallscale instabilities we also carried out a linear normalmode stability analysis by solving the Taylor-Goldstein equation,

$$
\phi^{\prime \prime}+\left[\frac{N^{2} k^{2}}{(k U-\omega)^{2}}-\frac{U^{\prime \prime}(z) k}{k U-\omega}-k^{2}\right] \phi=0,
$$

to obtain theoretical estimates of properties of unstable perturbations. For a spatial instability, the situation here, the frequency is real and given, whereas the wavenumber $k$ is the complex valued eigenvalue. The TaylorGoldstein equation is solved subject to homogeneous boundary conditions at $z=-100,0 \mathrm{~m}$. We calculated unstable eigenmodes using vertical profiles of the horizontal velocity and density at various locations in the ISWs. Results of the stability analysis, particularly for eigenmodes having wavelengths comparable to the ISW 
TABLE 3. Simulations using sinusoidal forcing. Case number refers to initial wave in Table 2. Here, $a$ and $\omega_{f}$ are the amplitude and frequency of the forcing, $-k_{i}$ and $k_{r}$ are the spatial growth rate and wavelength predicted by the Taylor-Goldstein equation at the center of the wave, and $-d E / d t$ is the rate energy is lost from the wave due to the energy flux associated with the billows.

\begin{tabular}{|c|c|c|c|c|c|c|}
\hline Case & $a\left(\mathrm{~m} \mathrm{~s}^{-2}\right)$ & $\omega_{f}\left(\mathrm{~s}^{-1}\right)$ & $-k_{i}\left(\mathrm{~m}^{-1}\right)$ & $k_{r}\left(\mathrm{~m}^{-1}\right)$ & $2 \pi / k_{r}(\mathrm{~m})$ & $-d E / d t\left(\mathrm{~W} \mathrm{~m}^{-1}\right)$ \\
\hline $1 \mathrm{a}$ & 0.0001 & 0.03 & 0.0158 & 0.061 & 103.2 & - \\
\hline $1 b$ & - & 0.05 & 0.0333 & 0.110 & 57.1 & 15 \\
\hline $1 \mathrm{c}$ & - & 0.06 & 0.0384 & 0.137 & 45.9 & 37 \\
\hline $1 d$ & - & 0.07 & 0.0414 & 0.164 & 38.3 & 48 \\
\hline $1 \mathrm{e}$ & - & 0.08 & 0.0431 & 0.190 & 33.1 & 45 \\
\hline 1f & - & 0.09 & 0.0436 & 0.216 & 29.1 & 40 \\
\hline $1 \mathrm{~g}$ & - & 0.10 & 0.0434 & 0.241 & 26.1 & 34 \\
\hline $1 \mathrm{~h}$ & - & 0.12 & 0.0409 & 0.290 & 21.7 & 23 \\
\hline $1 \mathrm{i}$ & - & 0.14 & 0.0367 & 0.336 & 18.7 & 5 \\
\hline $1 \mathrm{j}$ & - & 0.16 & 0.0312 & 0.381 & 16.5 & - \\
\hline $1 \mathrm{k}$ & 0.00005 & 0.10 & 0.0434 & 0.241 & 26.1 & - \\
\hline 11 & 0.0002 & 0.10 & - & - & - & 36 \\
\hline $1 \mathrm{~m}$ & 0.001 & 0.10 & - & - & - & 43 \\
\hline $2 \mathrm{a}$ & 0.0001 & 0.05 & 0.0317 & 0.115 & 54.6 & \\
\hline $2 b$ & - & 0.07 & 0.0394 & 0.169 & 37.2 & - \\
\hline $2 c$ & - & 0.08 & 0.0409 & 0.196 & 32.1 & - \\
\hline $2 \mathrm{~d}$ & - & 0.10 & 0.0411 & 0.248 & 25.3 & - \\
\hline $2 \mathrm{e}$ & - & 0.12 & 0.0387 & 0.297 & 21.2 & - \\
\hline $2 \mathrm{f}$ & - & 0.14 & 0.0346 & 0.345 & 18.2 & - \\
\hline $2 \mathrm{~g}$ & - & 0.16 & 0.0292 & 0.391 & 16.1 & - \\
\hline $3 a$ & - & 0.07 & 0.0347 & 0.177 & 35.5 & - \\
\hline $3 b$ & - & 0.08 & 0.0358 & 0.204 & 30.8 & - \\
\hline $3 c$ & - & 0.1 & 0.0353 & 0.257 & 24.4 & - \\
\hline
\end{tabular}

horizontal length scale, must be used with caution, because flow in an internal solitary wave is not horizontal as assumed in deriving the Taylor-Goldstein equation.

The results of this analysis are consistent with the numerical simulations (Figs. 15, 16). For the largest wave, the eigenvalue with the largest spatial growth rate at the wave center $\left(k=0.221-0.0436 i \mathrm{~m}^{-1}\right)$ occurs for $\omega_{f}=$ $0.092 \mathrm{~s}^{-1}$. In contrast, a temporal stability analysis predicts a maximum growth rate when $k=0.248 \mathrm{~m}^{-1}$, for which $\omega=0.103+0.0178 i \mathrm{~s}^{-1}$. We compared the predictions of linear stability theory with the results of the numerical simulations for case $1 \mathrm{~g}$ by extracting an isopycnal $z=\zeta(x, \sigma, t)$ from the upper sharp pycnocline $\left(\sigma=23.95 \mathrm{~kg} \mathrm{~m}^{-3}\right)$ and contrasting its displacement with the linear prediction. The perturbation of the isopycnal at $t_{0}=100 \mathrm{~min}$ was estimated by subtracting an estimate $\zeta\left(x-\delta c t_{0}, \sigma, 0\right)$ of its undisturbed height by taking into account the slow drift of the ISW at speed $\delta c$ (Table 1). Although this compensates for the estimated displacement of the wave by $1.8 \mathrm{~m}$ during the elapsed time, it does not compensate for possible changes in wave shape. Figure 17 compares the perturbation of the isopycnal $\delta \zeta$ with the shape of the isopycnal

$$
\delta \zeta_{t h}(x)=b+q \cos \left(k_{r} x+\alpha\right) e^{k_{i} x}
$$

predicted by the linear stability analysis, where $k_{r}$ and $k_{i}$ are given by the stability analysis, using velocity and density profiles at $x=40 \mathrm{~m}$, and $b, q$, and $\phi$ are chosen to match the isopycnal from the numerical simulation in the neighborhood of $x=40 \mathrm{~m}$. The stability analysis is in close agreement with both the wavelength and growth rate of the instability over the first wavelength of the perturbation. Thereafter, the wavelength and growth rate of the simulated perturbations increase, consistent with the stability analysis, which predicts smaller values of $k_{r}$ and larger growth rates $-k_{i}$ at the center of the wave.

Following previous authors, we also estimated the growth factor of a perturbation as it passes through the whole of the unstable region. Although other authors used a temporal stability analysis, our analysis is based

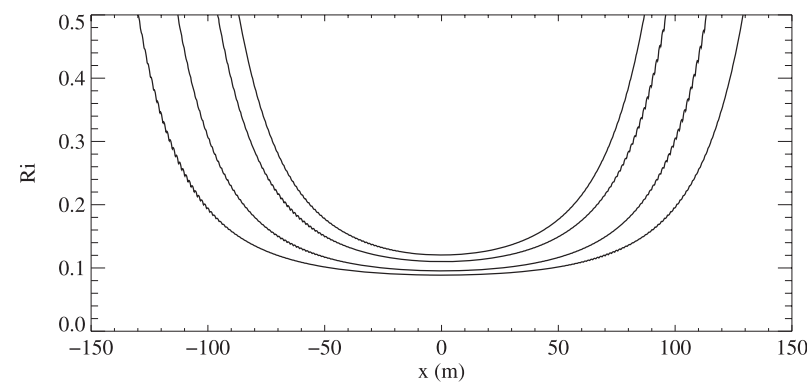

FIG. 12. Minimum Richardson number in the upper sharp pycnocline as a function of $x$ for initial waves 1-4 (see Table 2) with no background current and stratification with $\left(d_{1}, d_{2}\right)=(1.0,1.5) \mathrm{m}$. Richardson number decreases with wave amplitude. In each case, the center of the wave has been shifted to $x=0$. 

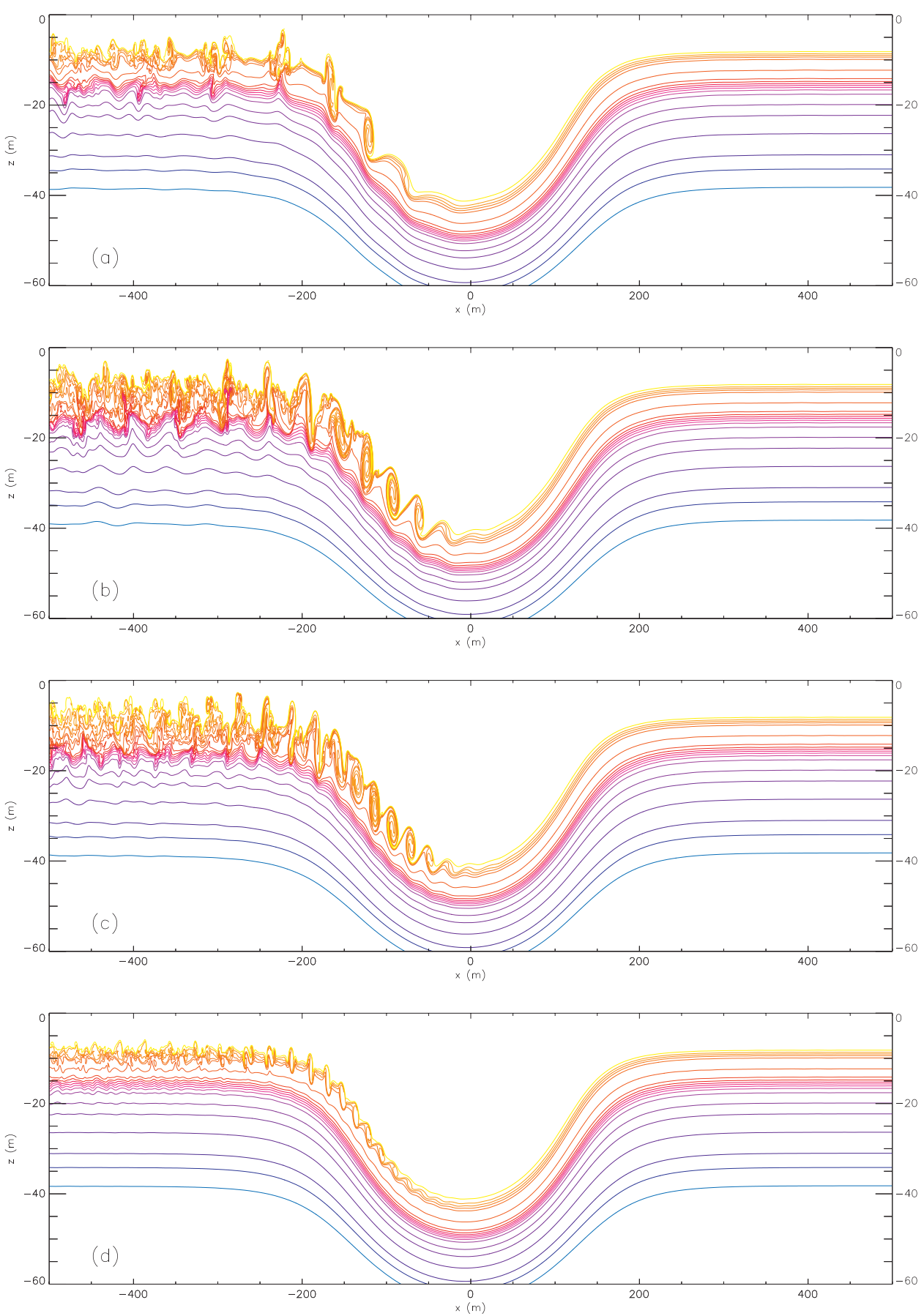

FIG. 13. Density contours 80 min after turning on the body forcing term. The initial wave is as in Fig. 10. Forcing amplitudes $a=0.0001 \mathrm{~m} \mathrm{~s}^{-2}$. (a) Case $1 \mathrm{~b}$ : forcing frequency $\omega_{f}=0.05 \mathrm{rad} \mathrm{s}^{-1}$. (b) Case $1 \mathrm{~d}: \omega_{f}=$ $0.07 \mathrm{rad} \mathrm{s}^{-1}$. (c) Case 1g: $\omega_{f}=0.1 \mathrm{rad} \mathrm{s}^{-1}$. (d) Case 1i: $\omega_{f}=0.14 \mathrm{rad} \mathrm{s}^{-1}$.

on a spatial stability analysis and we estimate the growth rate by integrating

$$
\frac{d a}{d x}=-k_{i}(x) a
$$

From this, we obtain the growth factor of the perturbation $a_{f} / a_{i}=e^{F}$, where $a_{i}$ and $a_{f}$ are the initial and final amplitudes of a perturbation passing through the region with $\mathrm{Ri}<0.25$. Values of $F=\ln \left(a_{f} / a_{i}\right)$ are included in Table 2. For the two strongly unstable waves, $F=5.7$ 

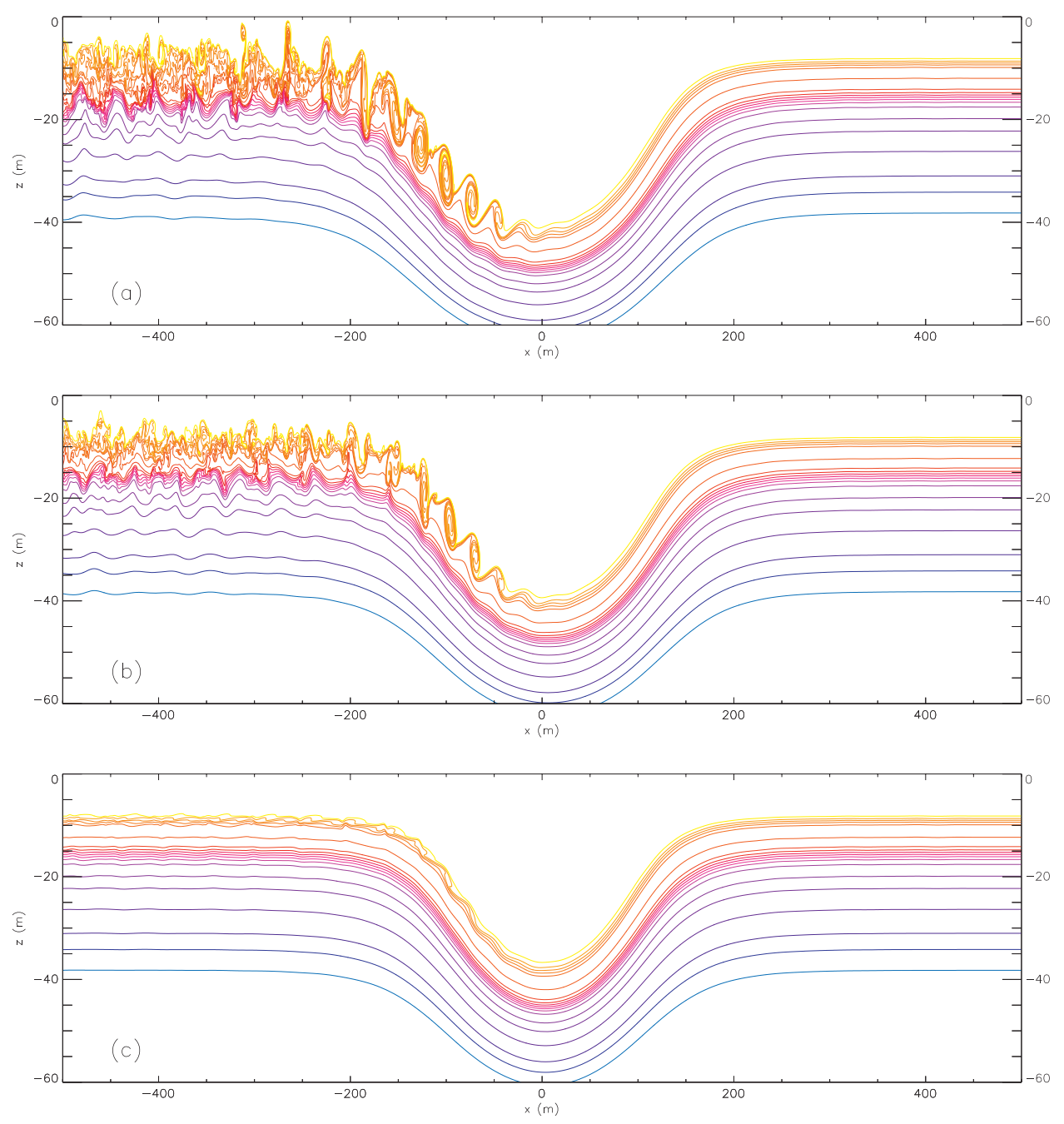

FIG. 14. Density contours $80 \mathrm{~min}$ after turning on the body forcing term for three different initial waves using a forcing frequency $\omega_{f}=0.08 \mathrm{rad} \mathrm{s}^{-1}$ and forcing amplitude $a=0.0001 \mathrm{~m} \mathrm{~s}^{-2}$. Cases (a) $1 \mathrm{e}$ (initial amplitude $33.9 \mathrm{~m}$ ), (b) $2 \mathrm{c}$ (initial amplitude $32.2 \mathrm{~m}$ ), and (c) $3 \mathrm{~b}$ (initial amplitude $29.8 \mathrm{~m}$ ).

and 7.0, whereas for the marginally unstable wave $F=3.8$. These values compare well with the findings of other authors, who used a more approximate temporal stability analysis: Troy and Koseff (2005) and Barad and Fringer (2010) found that instabilities occurred when their corresponding value of $F$, based on an average temporal growth rate, exceeded 5, whereas Fructus et al. (2009) found instabilities occurred when their value of $F$, based on maximum temporal growth rate, exceeded 5.4.

The top panel of Fig. 18 shows results for case $1 \mathrm{~m}\left(\omega_{f}=\right.$ $0.1 \mathrm{~s}^{-1}$ ), which has a forcing amplitude 10 times larger than that for case $1 \mathrm{~g}$ (see Fig. 13). The bottom two panels show results using the random forcing for $a_{\max }=10^{-6}$ and $10^{-4} \mathrm{~m} \mathrm{~s}^{-2}$. In both cases, irregularities in billows can be seen. For the randomly forced cases, the wavelengths of the perturbations in the center of the wave also vary with time.
For example, for the case using $a_{\max }=10^{-5} \mathrm{~m} \mathrm{~s}^{-2}$ (not shown), the wavelengths ranged between 20.2 and $29.2 \mathrm{~m}$, being generally at the longer end of this range. According to linear stability theory, these wavelengths correspond to forcing frequencies lying between 0.13 and $0.09 \mathrm{~s}^{-1}$.

Moum et al. (2003) performed a temporal stability analysis, using the Taylor-Goldstein equation, of a wave observed on the Oregon Shelf. As in the present analysis, the resolution and accuracy of velocity measurements within the range were severely limited and the density structure was used to compute streamlines. The horizontal velocity field at the center of the wave was derived from the inferred strain field and the observed velocity field ahead of the wave. Fructus et al. (2009) found good agreement between the linear stability analysis and the characteristics of the observed instabilities, 

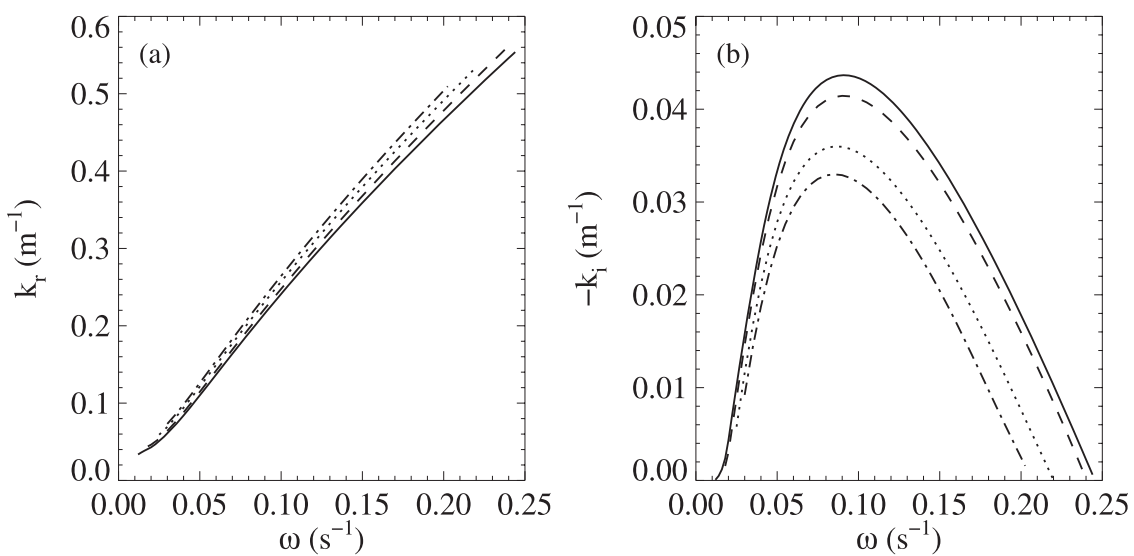

FIG. 15. Unstable eigenvalues as a function of the forcing frequency at center of the initial waves: (a) $k_{r}$ and (b) spatial growth rate $-k_{i}$. The lines are for case 1, 33.9-m wave (solid); case 2, 32.2-m wave (dashed); and case 3, 29.8-m wave (dotted).

with the theoretical growth rates of waves that broke being larger than those for waves that remained stable.

\section{Energetics}

As is fairly typical for a coastal wave, instabilities are an important part of the dissipation mechanism and the associated mixing plays a role in setting the stratification. Although these two-dimensional simulations cannot be used to address mixing, they can provide insight into the rate at which energy is lost from the ISW, because shear instabilities are initially two dimensional. As pointed out above, instabilities need time to grow. They also need time for three-dimensional effects to modify them and hence to affect the rate at which energy is extracted from the shear flow. To consider their role in extracting energy from the ISW, we consider the evolution of kinetic and

available potential energies and compare them with some observations to put our simulated values in context.

Neglecting viscous and diffusive effects, the pseudoenergy equation is

$$
\frac{\partial}{\partial t}\left(E_{k}+E_{a}\right)+\nabla \cdot\left[\mathbf{u}\left(E_{k}+E_{a}+p_{d}\right)\right]=0
$$

where $p_{d}$ is the pressure perturbation,

$$
E_{k}=\frac{\rho_{0}}{2}\left(u^{2}+w^{2}\right)
$$

is the total kinetic energy density, and

$$
E_{a}(x, z, t)=g \int_{z}^{z^{*}(x, z, t)}[\bar{\rho}(s)-\rho(x, z, t)] d s
$$
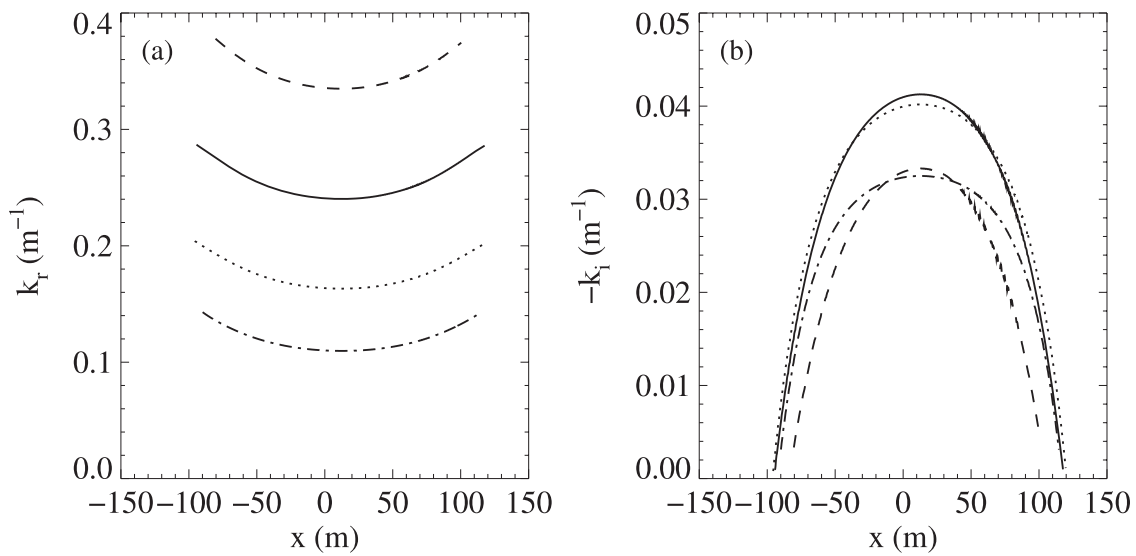

FIG. 16. Unstable eigenvalues as a function of $x$ for the 33.9-m wave (case 1): (a) $k_{r}$ and (b) spatial growth rate $-k_{i}$. The lines are $\omega_{f}=0.14 \mathrm{~s}^{-1}$ (dashed), $0.1 \mathrm{~s}^{-1}$ (solid), $0.07 \mathrm{~s}^{-1}$ (dotted), and $0.05 \mathrm{~s}^{-1}$ (dashed-dotted). 


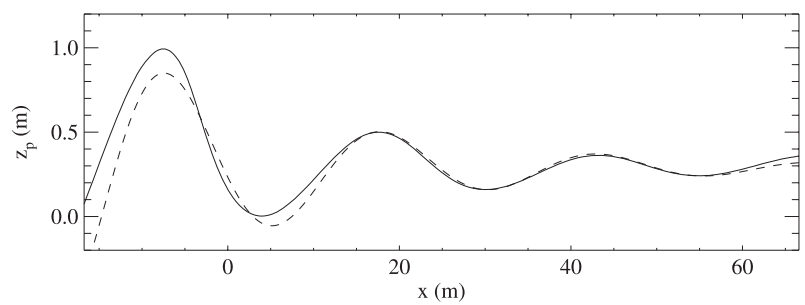

FIG. 17. Comparison of simulated isopycnal displacement with predictions of linear instability theory. Case 1g: the solid line is perturbation of $\sigma=23.95 \mathrm{~kg} \mathrm{~m}^{-3}$ isopycnal from displacement in initial wave at $t=100 \mathrm{~m}$, and the dashed line is theoretical isopycnal displacement using solution of TG equation at $x=40 \mathrm{~m}$. See text for more details.

is the available potential energy density. Here, $\bar{\rho}(z)$ is the reference density and $z^{*}(x, z, t)$ is the height of the fluid particle at $(x, z, t)$ in the reference stratification (Scotti et al. 2006; Lamb 2007, 2008; Lamb and Nguyen 2009). We use the background stratification as the reference density to calculate the APE in an infinitely long domain (Lamb 2008). The calculation of $E_{k}$ is done in a reference frame fixed with the fluid ahead of the wave.

The total energy in a region containing the solitary wave is $\bar{E}=\bar{K}+\overline{\mathrm{APE}}$, where $\bar{K}$ and $\overline{\mathrm{APE}}$ are the kinetic and available potential energy densities integrated over the region. Figure 19 shows the time evolution of the integrated energies, which include energy in both the ISW and the growing perturbations. The left panel compares $\bar{K}$ and $\overline{\mathrm{APE}}$, along with their average $\bar{E} / 2$, for case $1 \mathrm{~d}\left(\omega_{f}=0.07 \mathrm{~s}^{-1}\right)$. The kinetic energy is larger than the available potential energy (Turkington et al. 1991; Lamb and Nguyen 2009). As the small perturbations introduced by the forcing pass through the wave, they extract energy from the ISW and this energy is lost from the domain when the perturbations are advected out of it. When these enhanced perturbations start exiting the domain $(t \approx 1800 \mathrm{~s}), \bar{K}$ and $\overline{\mathrm{APE}}$ decay at the same rate. Figure $19 \mathrm{~b}$ compares the evolution of $\bar{E}$ for three simulations (cases 1d, $1 \mathrm{~g}$, and 1i) using a single forcing frequency $\left(\omega_{f}=0.07,0.1\right.$, and $\left.0.14 \mathrm{~s}^{-1}\right)$ and one case with random forcing $\left(a_{\max }=10^{-4} \mathrm{~m} \mathrm{~s}^{-2}\right)$. The energy initially decays slowly at $0.1 \mathrm{~W} \mathrm{~m}^{-1}$, a consequence of numerical dissipation, and then much more rapidly after the instabilities start removing energy from the domain. For the single frequency cases, the energy decreases linearly, whereas for the randomly forced case there is some variability about a mean linear decay rate. Final decay rates for several cases are given in Table 3. Interestingly, the largest decay rate $\left(48 \mathrm{~W} \mathrm{~m}^{-1}\right)$ occurs for a forcing frequency of $0.07 \mathrm{~s}^{-1}$, not for the frequency for which linear stability theory predicts the largest spatial growth rate. This could be due to differences in nonlinear growth rates of the $\mathrm{KH}$ billows and will be the subject of future work.

The decay rate also depends on the amplitude of the forcing function. For a forcing frequency of $0.1 \mathrm{~s}^{-1}$, increasing the amplitude of the forcing by a factor of 10 increases the decay rate by $26 \%$ from 34 to $43 \mathrm{~W} \mathrm{~m}^{-1}$ (cases $1 \mathrm{~g}$ and $1 \mathrm{~m}$ ), which is still smaller than the decay rate for $\sigma_{f}=0.07 \mathrm{~m} \mathrm{~s}^{-1}$ using the smaller forcing amplitude.

The initial wave energy is $4.24 \mathrm{MJ} \mathrm{m}^{-1}$ (MJ per meter crest length), which is typical for ISWs in shelf seas (Holloway 1987; Sandstrom et al. 1989; Jeans and Sherwin 2001). Moum et al. (2007) tracked a single ISW of depression as it traveled into shallow water on the Oregon Shelf in June 2000. Its energy decreased from 1 to $0.5 \mathrm{MJ} \mathrm{m}^{-1}$ as the depth decreased from 170 to $70 \mathrm{~m}$ over a distance of about $30 \mathrm{~km}$. The rate at which energy was lost was estimated to average about $10 \mathrm{~W} \mathrm{~m}^{-1}$ during this time.

For a decay rate of $50 \mathrm{~W} \mathrm{~m}^{-1}$, it would take our large wave $25 \mathrm{~h}$, traveling a distance of $56 \mathrm{~km}$ at $0.64 \mathrm{~m} \mathrm{~s}^{-1}$, to lose all of its energy. The decay rate, however, will decrease with time as the wave diminishes in amplitude, returning to small values when the wave restabilizes. Taking a typical length scale of an ISW as $L=600 \mathrm{~m}$, a decay rate of $50 \mathrm{~W} \mathrm{~m}^{-1}$ corresponds to an average depth-integrated decay rate of $(d \bar{E} / d t) / L=8 \times 10^{-2}$ $\mathrm{W} \mathrm{m}^{-2}$. As a comparison, Sandstrom and Elliott (1984) estimated a depth-integrated average decay rate of $5 \times 10^{-2} \mathrm{~W} \mathrm{~m}^{-2}$ for ISW packets on the Nova Scotia shelf and Jeans and Sherwin 2001 estimate values of $7.7 \times 10^{-2}$ decreasing to $2.1 \times 10^{-2} \mathrm{~W} \mathrm{~m}^{-2}$ for an ISW packet moving onto the Portuguese Shelf. Because these estimates are for wave packets, the estimated values will be lower than for an individual wave.

\section{Encounter with a surface pool of light fluid}

In the coastal environment, there can be considerable spatial inhomogeneity in the upper $5 \mathrm{~m}$ associated with freshwater runoff, in our case from the nearby Columbia River plume. Profiles taken ahead of the wave showed no sign of water with density less than $1024.25 \mathrm{~kg} \mathrm{~m}^{-3}$; however, it is clear from Fig. 2 that lower density water (both fresher and warmer) was present to a depth of almost $30 \mathrm{~m}$ in the center of the wave. Although the CTD measurements do not rule out the existence of a thin $(<2 \mathrm{~m})$ surface layer of lower density, there is the possibility of spatial heterogeneity in near-surface density structure close to the river plume. Although this is in no way surprising, the implications for the behavior of the ISW turn out to be very sensitive to variability in the 

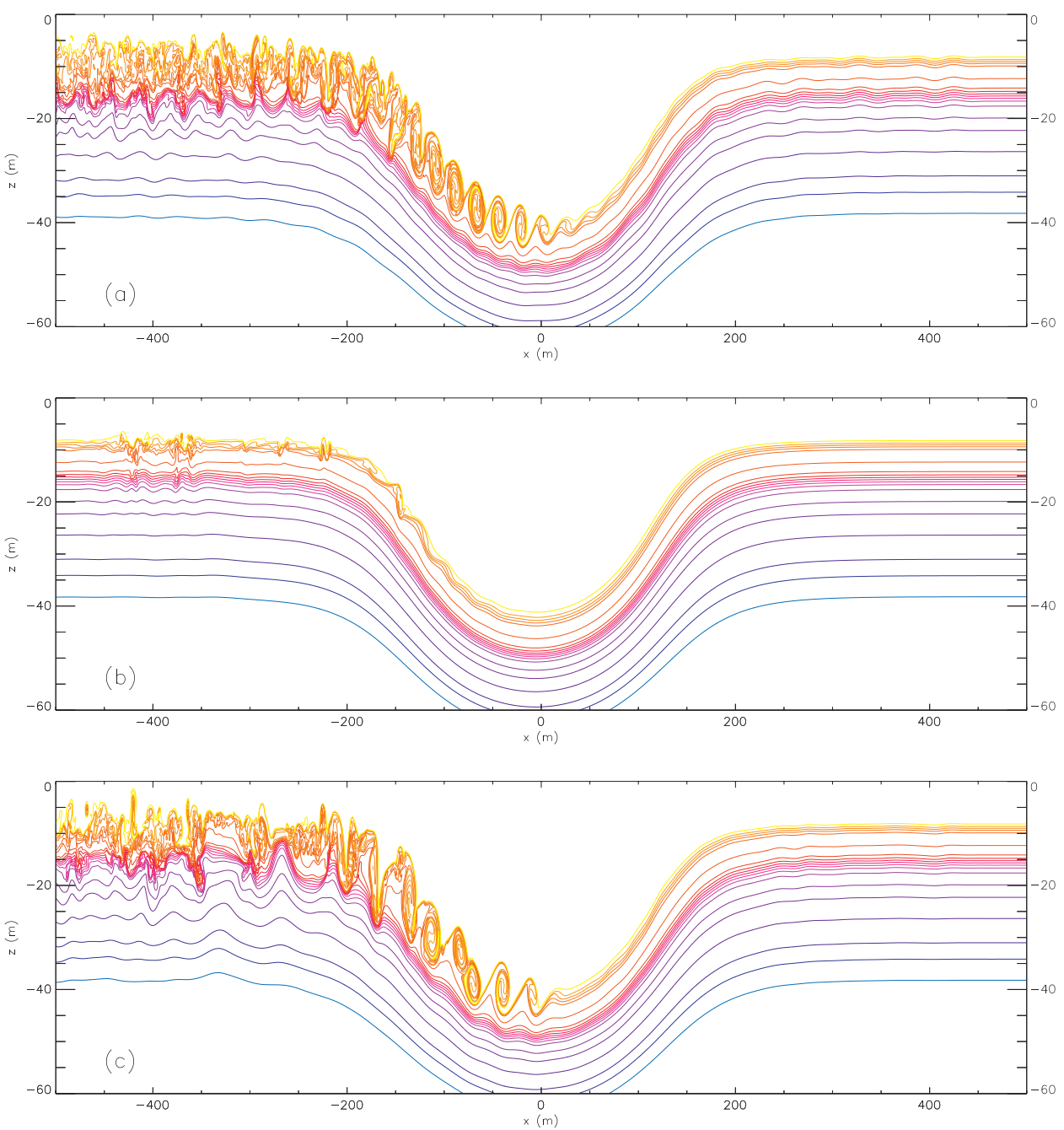

FIG. 18. Density contours $30 \mathrm{~min}$ after turning on the body forcing term using wave 1. (a) Sinusoidal forcing with $\omega_{f}=0.1 \mathrm{rad} \mathrm{s}^{-1}$ and forcing amplitude $a=0.001 \mathrm{~m} \mathrm{~s}^{-2}$. (b) Random forcing function with $a_{\max }=10^{-6} \mathrm{~m} \mathrm{~s}^{-2}$. (c) Random forcing function with $a_{\max }=10^{-4} \mathrm{~m} \mathrm{~s}^{-2}$.

near-surface water density if there is a background current with near-surface shear.

An ISW encountering a pool of light surface water was simulated for two shear profiles. The first was conducted using the setup from the previous section, namely an ISW of amplitude $34 \mathrm{~m}$ in the absence of a background current, and the second was conducted with a wave of similar amplitude $(36 \mathrm{~m})$ having a trapped core using background current $\bar{U}_{4}$.

For these simulations, the density at the inflow boundary was set to

$$
\begin{aligned}
\rho\left(x_{l}, z\right)= & \bar{\rho}(z)-F(t)\left[0.04\left(1+\tanh \frac{z+6}{2}\right)\right. \\
& \left.+0.06\left(1+\tanh \frac{z}{3}\right)\right],
\end{aligned}
$$

where

$$
F(t)=\frac{1}{4}\left(1+\tanh \frac{t-200.0}{100.0}\right)\left(1-\tanh \frac{t-3200}{100}\right) .
$$

Here, $t$ is time from the end of the adjustment time of the initial wave. Fluid flowing through the right boundary then advects this light fluid into the wave. The surface pool, with total length of about $1.7 \mathrm{~km}$, lies above the pycnocline in the upper $6 \mathrm{~m}$ of the water column. It has a density of $\sigma=24.1 \mathrm{~kg} \mathrm{~m}^{-3}$ at the surface compared with $24.24 \mathrm{~kg} \mathrm{~m}^{-3}$ for the initial density and the minimum of $24.15 \mathrm{~kg} \mathrm{~m}^{-3}$ observed in the wave.

The encounter of the wave without a trapped core (no background current) with the light surface water is 

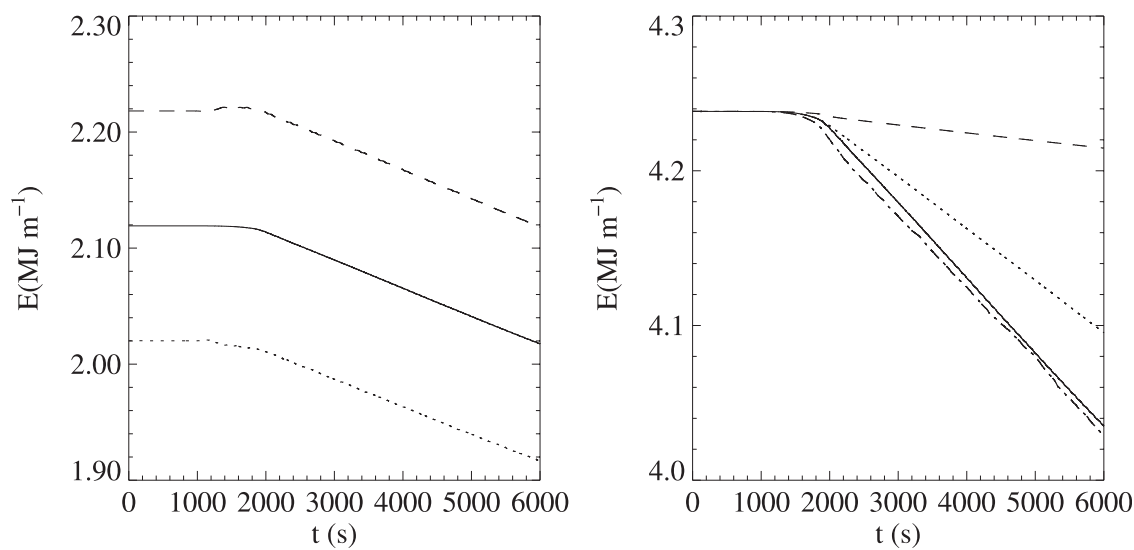

FIG. 19. Evolution of wave energy in computational domain ( $x$ between $\pm 500 \mathrm{~m}$ ). (a) Case 1d: Total kinetic energy (wave plus perturbation in reference frame fixed with fluid ahead of the wave; dashed curve), APE (dotted curve), and half the total energy (solid curve). (b) Total energy for cases $1 \mathrm{~d}, 1 \mathrm{~g}$, and $1 \mathrm{i}$ [ $\omega_{f}=0.07$ (solid curve), 0.1 (dotted curve), and $0.14 \mathrm{~s}^{-1}$ (dashed curve) for forcing amplitude $a=10^{-4} \mathrm{~m} \mathrm{~s}^{-2}$ ] and for the randomly forced case with $a_{\max }=$ $10^{-4} \mathrm{~m} \mathrm{~s}^{-2}$ (dashed-dotted curve).

illustrated in Fig. 20. The front of the light pool produces a disturbance in the pycnocline, which propagates ahead of the pool and becomes unstable in the center of the wave. This generates the large disturbance in the upper sharp pycnocline centered at about $x=$ $-350 \mathrm{~m}$ in Fig. 20a. Thereafter, no significant disturbance of the pycnocline occurs. The light surface pool thickens as it enters the wave, extending to a depth of over $30 \mathrm{~m}$. When its trailing edge is eventually advected out of the wave, shear instabilities form at its base (Fig. 20d), well above the pycnocline. These persist as the light water in the wave shrinks in volume and is eventually flushed out of the wave. There is some similarity with the observations (the high backscatter in the rear of the wave above the pycnocline in Fig. 2); however, the unstable region appears to be significantly smaller in the simulations.

Figure 21 shows the interaction of a pool of light surface water with an ISW with a core. At $t=13 \mathrm{~min}$ (Fig. 21a), the light surface water is beginning to enter the wave. Because of the presence of the wave core the surface fluid is prevented from sweeping across the top of the wave (Fig. 21b). Instead it starts to pass beneath the core, while pushing the core downstream (Fig. 21c). With light fluid beneath the denser fluid in the core, convective instabilities form and water is entrained into the core. Finally, the fluid in the core is replaced with somewhat lighter fluid (Fig. 21d). The core is now very active and exhibits features somewhat reminiscent of those seen in the field observations (Fig. 2); in particular, there is a large rollup near the back of the wave.

\section{Summary}

Observations of large-amplitude ISWs sometimes show striking overturning events. Here, we have selected a specific example to study with the goal of carrying out some numerical simulations to understand what can lead to such intermittent events, which include smallscale $\mathrm{KH}$ billows aligned with the pycnocline as well as much larger-scale overturns at the back of the wave above the pycnocline. The observations were acquired on the Oregon Shelf using a rapidly profiled CTD, a hull-mounted ADCP, and echo sounder imagery.

Using a nonhydrostatic two-dimensional numerical model, we first test the conditions required for development of shear flow instabilities in the sheared pycnocline beneath the core. An ISW comparable to the observed wave, in which shear instabilities could be simulated, required a wave that was larger than any that could be obtained as a direct solution of the DJL equation. We obtained such waves by first calculating a large-amplitude solution of the DJL equation for a different background current, after which the background current was changed and the wave was allowed to adjust to the new ambient conditions. Instabilities were then triggered by continuously driving perturbations in the pycnocline ahead of the wave, generally at a prescribed frequency. As the wave amplitude increases, the minimum Ri decreases and the length of the unstable patch increases. Both of these factors contribute to shear instability, and we found that instabilities occurred when $L_{x} / \lambda>0.8$, in good agreement with the experimental findings of Fructus et al. (2009), who found a critical value 

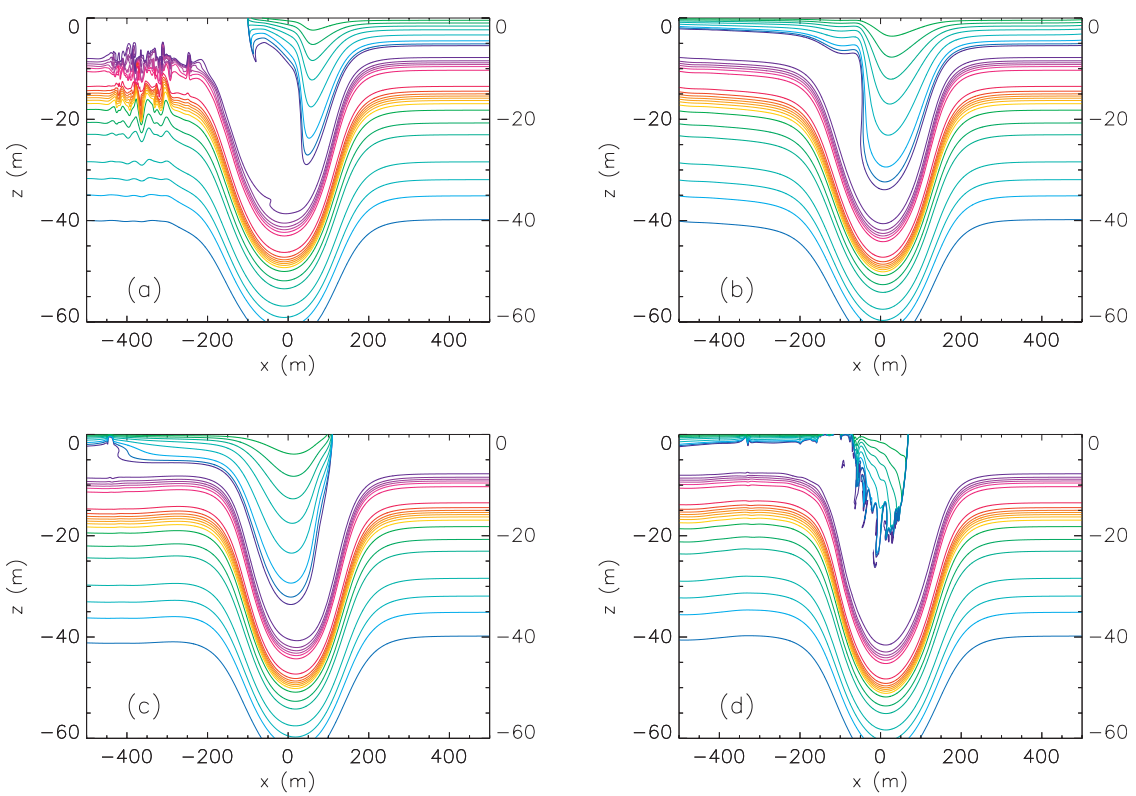

FIG. 20. Density contours showing the interaction of an ISW with a pool of light surface water. Initial wave with amplitude $34 \mathrm{~m}$ and no background current: (a) $t=30 \mathrm{~min}$, (b) $t=$ $50 \mathrm{~min}$, (c) $t=70 \mathrm{~min}$, and (d) $t=97 \mathrm{~min}$.

of 0.86 for a continuous three-layer stratification. The minimum Richardson number required for instabilities $(\mathrm{Ri}<0.11)$ is consistent with the findings of other authors (Troy and Koseff 2005; Fructus et al. 2009; Barad and Fringer 2010), as is the growth factor of the instabilities (about $e^{5.7}$ and $e^{7.0}$ for the largest two waves), based on a linear spatial instability analysis, as they pass through the low Richardson number region. The observations (see Fig. 2) suggest the presence of shear instabilities in both of the observed sharp pycnoclines. Simulation of shear instabilities in the lower sharp pycnocline could only be achieved by making the pycnocline much thinner than
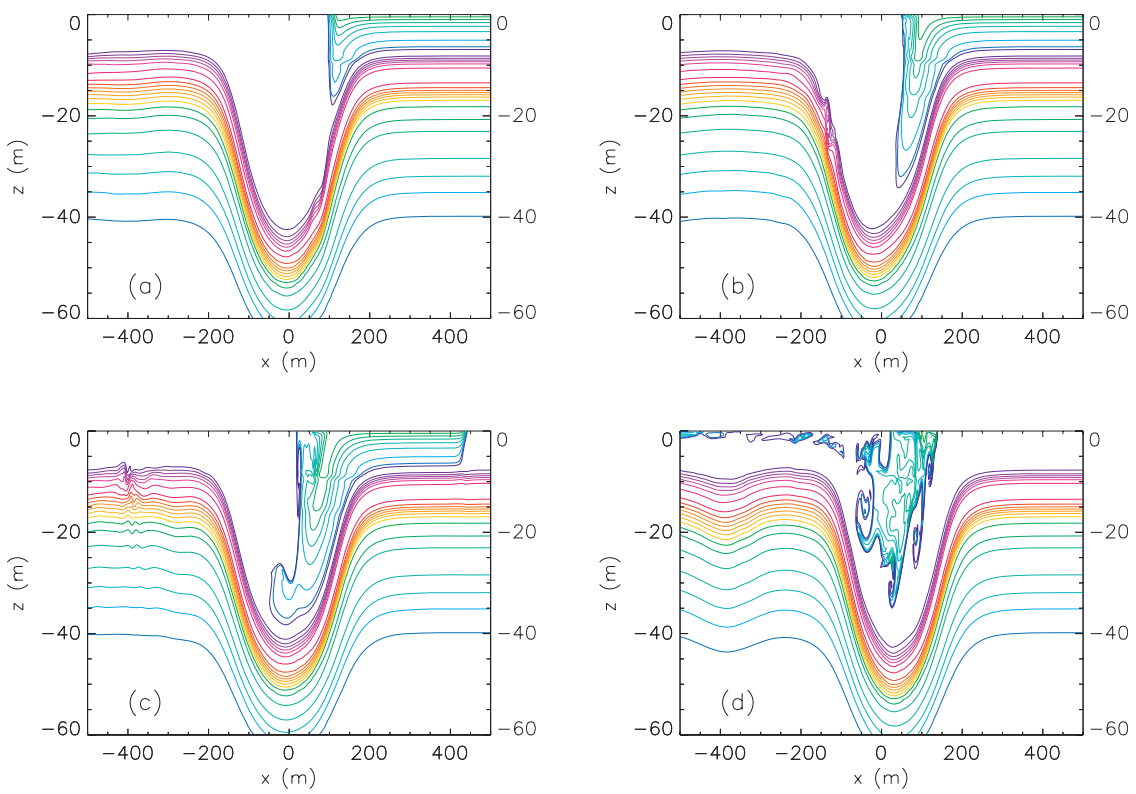

FIG. 21. Density contours showing the interaction of an ISW with a core with a pool of light surface water. Initial wave with amplitude $36 \mathrm{~m}$ and background velocity $U_{4}(z)$ : (a) $t=13 \mathrm{~min}$, (b) $t=20 \mathrm{~min}$, (c) $t=27 \mathrm{~min}$, and (d) $t=67 \mathrm{~min}$. 
observed or by adding strong background shear across it (results not discussed). Our simulated billows were up to $O(10)$ m thick, much larger than the observed overturns. These differences suggest that a finescale, unresolved structure in the background fields can have significant repercussions. The energy in the computed waves and the rate at which energy is drained from the wave by the shear instabilities are of the same order of magnitude as those inferred from observations in other coastal regions.

Because the observations detected the presence of light fluid in the upper $30 \mathrm{~m}$ of the wave, we investigated the interaction of an ISW with a localized pool of light surface water, which could be a natural consequence of the Columbia River plume. Two simulations of this type were done: one with and one without a trapped core. The presence of a trapped core required the use of a background current with near-surface shear having vorticity of the same sign as the wave-induced vorticity. Measurements from the ADCP begin at a depth of $8 \mathrm{~m}$, so we are unable to confirm the presence of such a background current. The wave with a trapped core encountering light surface fluid shows features similar to those in the trailing portion of the observed wave (Fig. 2). In contrast, our simulation of a wave encountering a pool of lower density surface water without a trapped core does not resemble the observations. The development of these features thus appears to require the presence of a trapped core in the ISW and a thin, localized surface layer of light fluid. Such patches are not unexpected in coastal environments with freshwater runoff and are consistent with observations ahead of and within the observed ISW. The combination of an active core and incorporation of a low-density surface layer results in complex patterns of convective instability, which appear consistent with our observations. The mechanism we propose for the unstable flow at the back of the wave (Fig. 2) differs from that hypothesized in Moum et al. (2003) to explain a different observation (their Fig. 14).

The numerical simulations also indicate a time scale for wave adjustment and entrainment of light fluid of about one hour, which is sufficiently long that waves on the Oregon Shelf never have time to fully adjust to their changing environment, a consequence of variable stratification and depth changes. We carried out a number of simulations of shoaling ISWs. The observed bottom slope is about 1:320. Simulations starting in depths of $140 \mathrm{~m}$ using bottom slopes as large as 1:80 were performed, and the waves were far from breaking at a depth of $100 \mathrm{~m}$, consistent with the observations. Shear instabilities appeared when the waves reached depths of about $65 \mathrm{~m}$, at which point the waves were highly asymmetric. We conclude that the observed overturning features are unlikely to be related to shoaling.
Care must be taken in interpreting our results because they are based on two-dimensional simulations. It is well known, however, that in parallel shear flows twodimensional perturbations have the largest growth rates and that as a consequence shear instabilities are initially two-dimensional but ultimately become three-dimensional (Barad and Fringer 2010). If the perturbations do not three-dimensionalize until after they leave the unstable patch where $\mathrm{Ri}<0.25$, their efficiency at extracting energy from the flow may be greatly diminished, in which case two-dimensional simulations may accurately predict both the occurrence of an instability as well as their form and the rate at which they extract energy from the ISW. Three-dimensional simulations are required before definitive conclusions can be made.

Acknowledgments. The observations described here were acquired as part of a collaboration with colleagues from Oregon State University; the Institute of Ocean Sciences, Sidney, British Columbia; and Scripps Institution of Oceanography. We are indebted to all participants as well as to the crew of the R/V Wecoma for their willing assistance in all phases of the project. Peter Chandler assisted with the data analysis. Jenny Lee assisted with the simulations and figures at the University of Waterloo. This work received support from the Office of Naval Research.

\section{REFERENCES}

Barad, M. F., and O. B. Fringer, 2010: Simulations of shear instabilities in interfacial gravity waves. J. Fluid Mech., 644, 61-95.

Bogucki, D., and C. Garrett, 1993: A simple model for the shearinduced decay of an internal solitary wave.J. Phys. Oceanogr., 23, 1767-1776.

__ , and L. G. Redekopp, 1999: A mechanism for sediment resuspension by internal solitary waves. Geophys. Res. Lett., 26 , 1317-1320.

_ and mixing by resonantly generated internal solitary waves. J. Phys. Oceanogr., 27, 1181-1196.

_ L. G. Redekopp, and J. Barth, 2005: Internal solitary waves in the Coastal Mixing and Optics 1996 experiment: Multimodal structure and resuspension. J. Geophys. Res., 110, C02024, doi:10.1029/2003JC002253.

Bourgault, D., and D. K. P. S. Galbraith, 2008: Turbulence and boluses on an internal beach. J. Mar. Res., 66, 563-588.

— M. D. Blokhina, R. Mirshak, and D. E. Kelley, 2007: Evolution of a shoaling internal solitary wavetrain. Geophys. Res. Lett., 34, L03601, doi:10.1029/2006GL028462.

Carr, M., D. Fructus, J. Grue, A. Jensen, and P. A. Davies, 2008: Convectively induced shear instability in large amplitude internal solitary waves. Phys. Fluids, 20, 126601, doi:10.1063/ 1.3030947.

Carter, G. S., M. C. Gregg, and R.-C. Lien, 2005: Internal waves, solitary-like waves, and mixing on the Monterey Bay shelf. Cont. Shelf Res., 25, 1499-1520.

Cummins, P. F., S. Vagle, L. Armi, and D. M. Farmer, 2003: Stratified flow over topography: Upstream influence and 
generation of nonlinear internal waves. Proc. Roy. Soc. London, 459A, 1467-1487.

Diamessis, P. J., and L. G. Redekopp, 2006: Numerical investigation of solitary internal wave-induced global instability in shallow water benthic boundary layers. J. Phys. Oceanogr., 36, 784-812.

Farmer, D. M., and J. D. Smith, 1980: Tidal interaction of stratified flow with a sill in knight inlet. Deep-Sea Res., A27, 239-254.

_ , Q. Li, and J.-H. Park, 2009: Internal wave observations in the South China Sea: The role of rotation and non-linearity. Atmos.-Ocean, 47, 267-280.

Fringer, O. B., and R. L. Street, 2003: The dynamics of breaking progressive interfacial waves. J. Fluid Mech., 494, 319-353.

Fructus, D., M. Carr, J. Grue, A. Jensen, and P. A. Davies, 2009: Shear-induced breaking of large internal solitary waves J. Fluid Mech., 620, 1-29, doi:10.1017/S0022112008004898.

Galbraith, P. S., and D. E. Kelley, 1996: Identifying overturns in CTD profiles. J. Atmos. Oceanic Technol., 13, 688-702.

Gerkema, T., 2001: Internal and interfacial tides: Beam scattering and local generation of solitary waves. J. Mar. Res., 59, 227-255.

Hogg, A. M., and G. N. Ivey, 2003: The Kelvin-Helmholtz to Holmboe instability transition in stratified exchange flows. J. Fluid Mech., 477, 339-363, doi:10.1017/S0022112002003397.

Holloway, P. E., 1987: Internal hydraulic jumps and solitons at a shelf break region on the Australian North West Shelf. J. Geophys. Res., 92, 5405-5416.

_ 1991: On dissipation of internal tides. Tidal Hydrodynamics, B. B. Parker, Ed., John Wiley and Sons, 449-466.

Hosegood, P., and H. van Haren, 2004: Near-bed solibores over the continental slope in the Faeroe-Shetland Channel. Deep-Sea Res. II, 51, 2943-2971.

Jeans, D. R. G., and T. J. Sherwin, 2001: The evolution and energetics of large amplitude nonlinear internal waves on the Portuguese shelf. J. Mar. Res., 59, 327-353.

Klymak, J. M., and J. N. Moum, 2003: Internal solitary waves of elevation advancing on a shoaling shelf. Geophys. Res. Lett., 30, 2045, doi:10.1029/2003GL017706.

Lamb, K. G., 1994: Numerical experiments of internal wave generation by strong tidal flow across a finite amplitude bank edge. J. Geophys. Res., 99, 848-864.

_ - 2002: A numerical investigation of solitary internal waves with trapped cores formed via shoaling. J. Fluid Mech., 451, 109-144.

_, 2003 : Shoaling solitary internal waves: On a criterion for the formation of waves with trapped cores. J. Fluid Mech., 478, 81-100.

_ 2007: Energy and pseudoenergy flux in the internal wave field generated by tidal flow over topography. Cont. Shelf Res., 27, 1208-1232.

- 2008: On the calculation of the available potential energy of an isolated perturbation in a density-stratified fluid. J. Fluid Mech., 597, 415-427, doi:10.1017/S0022112007009743.
— , and V. T. Nguyen, 2009: Calculating energy flux in internal solitary waves with an application to reflectance. J. Phys. Oceanogr., 39, 559-580.

Moum, J. N., D. M. Farmer, W. D. Smyth, L. Armi, and S. Vagle, 2003: Structure and generation of turbulence at interfaces strained by internal solitary waves propagating shoreward over the continental shelf. J. Phys. Oceanogr., 33, 2093-2112. ,,,--- , and — 2007: Dissipative losses in nonlinear internal waves propagating across the continental shelf. J. Phys. Oceanogr., 37, 1989-1995.

Nash, J. D., and J. N. Moum, 2005: River plumes as a source of large-amplitude internal waves in the coastal ocean. Nature, 437, 400-403, doi:10.1038/nature03936.

Orr, M. H., and P. C. Mignerey, 2003: Nonlinear internal waves in the South China Sea: Observation of the conversion of depression internal waves to elevation internal waves. J. Geophys. Res., 108, 3064, doi:10.1029/2001JC001163.

Osborne, A. R., and T. L. Burch, 1980: Internal solitons in the Andaman Sea. Science, 208, 451-460.

Sandstrom, H., and J. A. Elliott, 1984: Internal tide and solitons on the Scotian Shelf: A nutrient pump at work. J. Geophys. Res., 89, 6415-6426.

,-- , and N. A. Cochrane, 1989: Observing groups of solitary internal waves and turbulence with BATFISH and echosounder. J. Phys. Oceanogr., 19, 987-997.

Scotti, A., R. Beardsley, and B. Butman, 2006: On the interpretation of energy and energy fluxes of nonlinear internal waves: An example from Massachusetts Bay. J. Fluid Mech., 561, 103-112.

Stastna, M., and K. G. Lamb, 2002: Vortex shedding and sediment resuspension associated with the interaction of an internal solitary wave and the bottom boundary layer. Geophys. Res. Lett., 29, 1512, doi:10.1029/2001GL014070.

_ _, and W. R. Peltier, 2004: Upstream-propagating solitary waves and forced internal-wave breaking in stratified flow over a sill. Proc. Roy. Soc., 460A, 3159-3190.

_ , and K. G. Lamb, 2008: Sediment resuspension mechanisms associated with internal waves in coastal waters. J. Geophys. Res., 113, C10016, doi:10.1029/2007JC004711.

Thorpe, S. A., 1968: On the shape of progressive internal waves. Philos. Trans. Roy. Soc. London, 263A, 563-614.

Troy, C. D., and J. R. Koseff, 2005: The instability and breaking of long internal waves. J. Fluid Mech., 543, 107-136, doi:10.1017/ S0022112005006798.

Turkington, B., A. Eydeland, and S. Wang, 1991: A computational method for solitary internal waves in a continuously stratified fluid. Stud. Appl. Math., 85, 93-127.

Vlasenko, V. I., and K. Hutter, 2002: Transformation and disintegration of strongly nonlinear internal waves by topography in stratified lakes. Ann. Geophys., 20, 2087-2103.

Woods, J. D., 1968: Wave induced shear instability in the summer thermocline. J. Fluid Mech., 32, 791-800. 